\title{
The Weinstein conjecture for stable Hamiltonian structures
}

\author{
Michael Hutchings \\ CLIFFORd HENRY TAUbes
}

\begin{abstract}
We use the equivalence between embedded contact homology and Seiberg-Witten Floer homology to obtain the following improvements on the Weinstein conjecture. Let $Y$ be a closed oriented connected 3-manifold with a stable Hamiltonian structure, and let $R$ denote the associated Reeb vector field on $Y$. We prove that if $Y$ is not a $T^{2}$-bundle over $S^{1}$, then $R$ has a closed orbit. Along the way we prove that if $Y$ is a closed oriented connected 3-manifold with a contact form such that all Reeb orbits are nondegenerate and elliptic, then $Y$ is a lens space. Related arguments show that if $Y$ is a closed oriented 3-manifold with a contact form such that all Reeb orbits are nondegenerate, and if $Y$ is not a lens space, then there exist at least three distinct embedded Reeb orbits.
\end{abstract}

57R17, 57R57, 53D40; 57R58

\section{Introduction}

Throughout this paper, $Y$ denotes a closed, oriented, connected 3-manifold. Recall that a contact form on $Y$ is a $1-$ form $\lambda$ on $Y$ such that $\lambda \wedge d \lambda>0$. A contact form $\lambda$ determines a contact structure, namely the two-plane field $\xi:=\operatorname{Ker}(\lambda)$. It also determines a vector field $R$, called the Reeb vector field, characterized by $d \lambda(R, \cdot)=0$ and $\lambda(R)=1$. A Reeb orbit is a closed orbit of $R$, ie a map $\gamma: \mathbb{R} / T \mathbb{Z} \rightarrow Y$ for some $T>0$ such that $\gamma^{\prime}(t)=R(\gamma(t))$. Two Reeb orbits are considered equivalent if they differ by precomposition with a translation of $\mathbb{R} / T \mathbb{Z}$.

The three-dimensional version of the Weinstein conjecture asserts that for every closed oriented 3-manifold $Y$, and for every contact form $\lambda$ on $Y$, there exists a Reeb orbit. There is a long history of work proving this conjecture in many cases, for example for overtwisted contact structures by Hofer [8], for contact structures supported by planar open books by Abbas, Cieliebak and Hofer [1] and for many additional types of open books by Colin and Honda [5]. The Weinstein conjecture was recently proved by the second author in all cases in [23].

In fact a stronger result is proved by the second author in [24], which asserts that a version of the Seiberg-Witten Floer homology of $-Y$ as defined by Kronheimer and 
Mrowka [19], namely the version $\widetilde{H M}(-Y)$, is isomorphic to the embedded contact homology $(\mathrm{ECH})$ of $(Y, \lambda)$. To see how this implies the Weinstein conjecture, recall that the $\mathrm{ECH}$ of $(Y, \lambda)$ is the homology of a chain complex which is generated by certain unions of Reeb orbits with multiplicities and whose differential counts certain embedded holomorphic curves in $\mathbb{R} \times Y$. If $Y$ has a contact form with no Reeb orbit, then the ECH has just one generator, namely the empty set of Reeb orbits. However Kronheimer and Mrowka have shown that $\widehat{H M}$ of every closed oriented 3-manifold is infinitely generated [19]. Together with the isomorphism between ECH and $\widetilde{H M}$, this gives a contradiction, and the Weinstein conjecture is proved.

The original proof of the Weinstein conjecture in [23] established and used only a first step towards the isomorphism between $\mathrm{ECH}$ and $\widetilde{H M}$, which entailed showing how generators of $\widetilde{H M}$ give rise to generators of $\mathrm{ECH}$. In the present paper, we exploit the full strength of the isomorphism to obtain two improvements on the Weinstein conjecture. First, we extend the class of vector fields for which a closed orbit is known to exist. Second, in most cases we can increase the lower bound on the number of (embedded) Reeb orbits from one to three.

To explain the first improvement: A contact form is a special case of a stable Hamiltonian structure, a notion which was identified by Bourgeois et al [3] and Cieliebak and Mohnke [4] as a general setting in which one can obtain Gromov-type compactness for moduli spaces of holomorphic curves in $\mathbb{R} \times Y$. If $Y$ is an oriented three-manifold, a stable Hamiltonian structure on $Y$ is a pair $(\lambda, \omega)$, where $\lambda$ is a 1 -form on $Y$ and $\omega$ is a $2-$ form on $Y$, such that

$$
\begin{gathered}
d \omega=0, \\
\lambda \wedge \omega>0, \\
\operatorname{Ker}(\omega) \subset \operatorname{Ker}(d \lambda) .
\end{gathered}
$$

Note that the second condition implies that $\omega$ is nonvanishing, and consequently the third condition is equivalent to

$$
d \lambda=f \omega
$$

where $f: Y \rightarrow \mathbb{R}$ is a smooth function.

A stable Hamiltonian structure determines a vector field $R$ on $Y$, which we call the Reeb vector field, characterized by

$$
\omega(R, \cdot)=0, \quad \lambda(R)=1 .
$$

It follows from the definitions that the stable Hamiltonian structure is invariant under $R$ :

$$
\mathcal{L}_{R} \lambda=0, \quad \mathcal{L}_{R} \omega=0, \quad \mathcal{L}_{R} f=0 .
$$


Example A contact 1 -form $\lambda$ determines a stable Hamiltonian structure in which $\omega=d \lambda$ and $R$ is the Reeb vector field in the usual sense. Here $f \equiv 1$.

Example Let $\Sigma$ be a closed oriented surface with a symplectic form $\omega$, and let $\phi$ be a symplectomorphism of $(\Sigma, \omega)$. Let $Y$ be the mapping torus

$$
Y:=\frac{[0,1] \times \Sigma}{(1, x) \sim(0, \phi(x))} .
$$

This fibers over $S^{1}=\mathbb{R} / \mathbb{Z}$. Let $R$ denote the vector field on $Y$ which increases the $[0,1]$ coordinate in (1-2). Note that closed orbits of $R$ correspond to periodic orbits of $\phi$. Now $Y$ has a stable Hamiltonian structure in which $R$ is as described above, $\lambda$ is the pullback of the volume form on $S^{1}$, and $\omega$ is the unique extension of the closed 2 -form on the fibers of $Y \rightarrow S^{1}$ to a 2 -form on $Y$ which annihilates $R$. Here $f \equiv 0$.

Stable Hamiltonian structures are also carried by "stable hypersurfaces" in symplectic manifolds; see Cieliebak and Mohnke [4, Section 2.3]. The Weinstein conjecture is known to hold for compact stable hypersurfaces in $\mathbb{R}^{2 n}$; see Hofer and Zehnder [12, Section 4.3]. This suggests that in three dimensions it might be possible to generalize the Weinstein conjecture from contact structures to stable Hamiltonian structures. The main result of the present paper is that this is almost always true:

Theorem 1.1 Let $Y$ be a closed oriented connected 3-manifold with a stable Hamiltonian structure. If $Y$ is not a $T^{2}$-bundle over $S^{1}$, then the associated Reeb vector field has a closed orbit.

Note that there exist $T^{2}$-bundles over $S^{1}$ with stable Hamiltonian structures having no Reeb orbit. Examples with $f=0$ are provided by the mapping tori of symplectomorphisms $\phi: T^{2} \rightarrow T^{2}$ with no periodic orbit. Here $\phi$ can be an irrational rotation, or an appropriate composition of an irrational rotation with a Dehn twist. In addition, the construction in Section 5 can be inverted to produce examples of $T^{2}$-bundles over $S^{1}$ with stable Hamiltonian structures in which $f$ changes sign and there is no Reeb orbit.

The outline of the proof of Theorem 1.1 is as follows. Assume that $R$ has no closed orbit. By the Weinstein conjecture, $f$ is sometimes zero; and if $f$ is identically zero then $Y$ fibers over $S^{1}$ and a calculation using the Lefschetz fixed point theorem shows that the fiber is $T^{2}$. So assume that $f$ is sometimes zero and sometimes nonzero. Choose $\varepsilon>0$ small such that both $\varepsilon$ and $-\varepsilon$ are regular values of $f$. Since $f$ is 
invariant under the nonvanishing vector field $R$, the level sets $f^{-1}( \pm \varepsilon)$ are disjoint unions of tori. We can now decompose $Y$ along a union of tori as

$$
Y=f^{-1}(-\infty,-\varepsilon] \cup f^{-1}[-\varepsilon, \varepsilon] \cup f^{-1}[\varepsilon, \infty) .
$$

It is enough to show that each piece in the decomposition is a disjoint union of copies of $T^{2} \times I$.

To do this for the middle piece $f^{-1}[-\varepsilon, \varepsilon]$, the idea is to use the fact that $f$ is close to zero to show that the middle piece fibers over $S^{1}$. We then use the assumption that there are no Reeb orbits to show that the fibers are disjoint unions of annuli. This part of the argument does not use any Floer homology.

The remaining pieces in the decomposition can be collapsed into closed contact manifolds with each boundary torus becoming an elliptic Reeb orbit. To conclude the proof of Theorem 1.1, we then invoke the following theorem. To state it, recall that a Reeb orbit $\gamma$ is called nondegenerate if its linearized return map, ie the symplectic linear map from $\xi_{\gamma(0)}$ to itself given by the linearized Reeb flow along $\gamma$, does not have 1 as an eigenvalue. We usually assume that all Reeb orbits (including multiple covers) are nondegenerate. In this case one can then classify the Reeb orbits into three types according to the eigenvalues $\lambda, \lambda^{-1}$ of the linearized return map:

- elliptic: $\lambda, \lambda^{-1}$ on the unit circle.

- positive hyperbolic: $\lambda, \lambda^{-1}>0$.

- negative hyperbolic: $\lambda, \lambda^{-1}<0$.

Theorem 1.2 Let $Y$ be a closed oriented connected 3-manifold with a contact form $\lambda$ such that all Reeb orbits are nondegenerate and elliptic. Then $Y$ is a lens space, there are exactly two embedded Reeb orbits, and they are the core circles in the solid tori of a genus one Heegaard splitting of $Y$.

The proof of Theorem 1.2 extensively uses the isomorphism between ECH and $\widetilde{H M}$. The outline is as follows. If all Reeb orbits are elliptic, then the differential on the ECH chain complex vanishes, because all generators have even grading. The isomorphism with $\widetilde{H M}$ then implies that all Reeb orbits represent torsion homology classes. If the number of embedded Reeb orbits is less than or greater than two, then the growth rate of the number of nullhomologous ECH generators with grading $\leq I$ as $I$ goes to infinity is either too slow or too fast to be consistent with known properties of $\widetilde{H M}$. Next, the equivalence of the $U$ maps in the two theories guarantees the existence of many holomorphic curves between the ECH generators. Finally, a probabilistic argument shows that at least one of these holomorphic curves is in fact a cylinder whose 
projection to $Y$ is an embedding. This gives rise to a foliation of $Y$ by holomorphic cylinders with boundary on the two Reeb orbits, and this foliation yields the desired Heegaard splitting.

A corollary of Theorem 1.2 is that it is impossible for there to exist only one embedded Reeb orbit and for that orbit to be nondegenerate and elliptic. Using $\mathrm{ECH}=\widetilde{H M}$ a bit more, one can upgrade this to show:

Theorem 1.3 Let $Y$ be a closed oriented 3-manifold with a contact form such that all Reeb orbits are nondegenerate. Then there are at least two distinct embedded Reeb orbits; and if $Y$ is not a lens space then there are at least three distinct embedded Reeb orbits.

There is certainly room for improvement on the lower bound in Theorem 1.3, at least if one knows more about the three-manifold $Y$ and its contact structure. In fact, work of Colin and Honda [5] using linearized contact homology shows that many threemanifolds with contact structures have the property that for any contact form there must be infinitely many distinct embedded Reeb orbits.

Update After posting the first version of this paper, we received a draft of the thesis of Ana Rechtman [20], which contains constructions that are relevant to Theorem 1.1.

The rest of the paper In Section 2 we review the basics of ECH. In Section 3 we discuss some more subtle aspects of ECH that we will need. In Section 4 we prove Theorem 1.2 regarding contact manifolds with all Reeb orbits elliptic, and we also prove the lower bound in Theorem 1.3. In Section 5 we prove the main Theorem 1.1.

Acknowledgements The first author was partially supported by NSF grant DMS0505884. The second author was partially supported by the National Science Foundation.

\section{Review of embedded contact homology}

We now review the basic notions from embedded contact homology $(\mathrm{ECH})$ that will be needed in the present paper. References are Hutchings $[13 ; 14]$ for the ECH index theory, Hutchings and Sullivan [16] for additional structure on ECH and Hutchings and Taubes [17] for the analysis. Below, fix a closed oriented connected 3-manifold $Y$ as usual, and fix a contact form $\lambda$ on $Y$ such that all Reeb orbits are nondegenerate. 


\subsection{ECH generators}

Definition 2.1 An ECH generator is a finite set of pairs $\alpha=\left\{\left(\alpha_{i}, m_{i}\right)\right\}$ such that the $\alpha_{i}$ 's are distinct embedded Reeb orbits, the $m_{i}$ 's are positive integers and $m_{i}=1$ whenever $\alpha_{i}$ is hyperbolic. The homology class of $\alpha$ is defined to be

$$
[\alpha]:=\sum_{i} m_{i}\left[\alpha_{i}\right] \in H_{1}(Y)
$$

For each $\Gamma \in H_{1}(Y)$, the embedded contact homology $\mathrm{ECH}_{*}(Y, \lambda, \Gamma)$ is the homology of a chain complex $C_{*}(Y, \lambda, \Gamma)$ which is freely generated over $\mathbb{Z}$ by the $\mathrm{ECH}$ generators $\alpha$ with $[\alpha]=\Gamma$.

Before defining the grading and the differential on this chain complex, we make two remarks. First, the empty set $\alpha=\phi$ is a legitimate ECH generator with $[\alpha]=0$. In fact the $\mathrm{ECH}$ generator $\phi$ turns out to be a cycle in the $\mathrm{ECH}$ chain complex $C_{*}(Y, \lambda, 0)$, whose homology class in ECH conjecturally agrees with the contact invariant in $\widetilde{H M}$. Second, we sometimes write an ECH generator $\alpha=\left\{\left(\alpha_{i}, m_{i}\right)\right\}$ using the multiplicative notation $\prod_{i} \alpha_{i}^{m_{i}}$. However the grading and differential on the ECH chain complex do not behave simply with respect to this sort of multiplication.

\subsection{The ECH index}

We now explain the grading on the chain complex.

Notation 2.2 If $\alpha=\left\{\left(\alpha_{i}, m_{i}\right)\right\}$ and $\beta=\left\{\left(\beta_{j}, n_{j}\right)\right\}$ are two ECH generators with $[\alpha]=[\beta]=\Gamma$, define $H_{2}(Y, \alpha, \beta)$ to be the set of equivalence classes of 2-chains $Z$ in $Y$ with

$$
\partial Z=\sum_{i} m_{i} \alpha_{i}-\sum_{j} n_{j} \beta_{j}
$$

where two such 2-chains are considered equivalent if they differ by the boundary of a $3-$ chain. Thus $H_{2}(Y, \alpha, \beta)$ is an affine space over $H_{2}(Y)$.

Definition 2.3 If $Z \in H_{2}(Y, \alpha, \beta)$, define the $E C H$ index

$$
I(\alpha, \beta, Z):=c_{\tau}(Z)+Q_{\tau}(Z)+\sum_{i} \sum_{k=1}^{m_{i}} \mathrm{CZ}_{\tau}\left(\alpha_{i}^{k}\right)-\sum_{j} \sum_{k=1}^{n_{j}} \mathrm{CZ}_{\tau}\left(\beta_{j}^{k}\right) \in \mathbb{Z} .
$$

Here $\tau$ is a trivialization of the contact plane field $\xi$ over the $\alpha_{i}$ 's and $\beta_{j}$ 's; $c_{\tau}(Z)$ denotes the relative first Chern class of $\xi$ over $Z$ with respect to the boundary trivialization $\tau ; Q_{\tau}(Z)$ denotes the relative intersection pairing; $\gamma^{k}$ denotes the $k$-fold 
iterate of $\gamma$, ie the pullback of $\gamma: \mathbb{R} / T \mathbb{Z} \rightarrow Y$ to $\mathbb{R} / k T \mathbb{Z}$; and $\mathrm{CZ}_{\tau}$ denotes the Conley-Zehnder index. For the detailed definitions of the integers $c_{\tau}(Z), Q_{\tau}(Z)$ and $\mathrm{CZ}_{\tau}\left(\gamma^{k}\right)$, see Hutchings $[13 ; 14]$.

As explained in $[13 ; 14]$, the ECH index $I(\alpha, \beta, Z)$ does not depend on the choice of trivialization $\tau$, even though the individual terms in its definition do. It does depend on $Z$ : if $Z^{\prime} \in H_{2}(Y, \alpha, \beta)$ is another relative homology class, then by [13, Equation (5)] and [13, Lemma 2.5(a)] we have

$$
\begin{aligned}
c_{\tau}(Z)-c_{\tau}\left(Z^{\prime}\right) & =\left\langle c_{1}(\xi), Z-Z^{\prime}\right\rangle, \\
Q_{\tau}(Z)-Q_{\tau}\left(Z^{\prime}\right) & =2 \Gamma \cdot\left(Z-Z^{\prime}\right) .
\end{aligned}
$$

Therefore

$$
I(\alpha, \beta, Z)-I\left(\alpha, \beta, Z^{\prime}\right)=\left\langle c_{1}(\xi)+2 \operatorname{PD}(\Gamma), Z-Z^{\prime}\right\rangle .
$$

Consequently, if $[\alpha]=[\beta]=\Gamma$, then it makes sense to define

$$
I(\alpha, \beta):=I(\alpha, \beta, Z) \in \mathbb{Z} / d_{\Gamma},
$$

where $Z$ is any element of $H_{2}(Y, \alpha, \beta)$, and $d_{\Gamma}$ denotes the divisibility of $c_{1}(\xi)+$ $2 \mathrm{PD}(\Gamma)$ in $H^{2}(Y ; \mathbb{Z})$ mod torsion. Note that $d_{\Gamma}$ is an even integer.

It is also shown in [13, Proposition 1.6(b)] that $I$ is additive in the following sense: If $\gamma$ is another ECH generator with $[\gamma]=\Gamma$ and if $W \in H_{2}(Y, \beta, \gamma)$, then $Z+W \in$ $\mathrm{H}_{2}(Y, \alpha, \gamma)$ is defined and

$$
I(\alpha, \gamma, Z+W)=I(\alpha, \beta, Z)+I(\beta, \gamma, W) .
$$

It follows that (2-3) defines a relative $\mathbb{Z} / d_{\Gamma}$ grading on the chain complex $C_{*}(Y, \lambda, \Gamma)$.

It is further shown in [14] that the relative grading (2-3) can be refined to an absolute grading which associates to each ECH generator a homotopy class of oriented 2-plane fields on $Y$. In the present paper we will not need this absolute grading and can just regard the grading on $C_{*}(Y, \lambda, \Gamma)$ as taking values in some abstract affine space over $\mathbb{Z} / d_{\Gamma}$. However we do need to know, from [13, Proposition 1.6(c)], that the mod 2 grading is given by

$$
I(\alpha, \beta) \equiv I_{2}(\alpha)-I_{2}(\beta) \in \mathbb{Z} / 2 .
$$

Here if $\alpha=\left\{\left(\alpha_{i}, m_{i}\right)\right\}$ is an ECH generator, then $I_{2}(\alpha) \in \mathbb{Z} / 2$ denotes the mod 2 count of orbits $\alpha_{i}$ that are positive hyperbolic. 


\subsection{The index inequality}

To prepare to define the differential on the ECH chain complex, choose an almost complex structure $J$ on $\mathbb{R} \times Y$ satisfying the following properties. Let $s$ denote the $\mathbb{R}$ coordinate on $\mathbb{R} \times Y$, and recall that $R$ denotes the Reeb vector field. We require that $J(\partial / \partial s)=R$, that $J$ is invariant under the map $(s, y) \mapsto(s+c, y)$ for each $c \in \mathbb{R}$, and that $J$ sends the contact plane field $\xi$ to itself, rotating positively in the sense that $d \lambda(v, J v) \geq 0$ for all $v \in \xi$.

We consider holomorphic curves $u:(C, j) \rightarrow(\mathbb{R} \times Y, J)$ such that the domain $C$ is a punctured compact Riemann surface, and $u$ is not constant on any component of $C$. The domain $C$ is not required to be connected. We often abuse notation and denote the holomorphic curve $u$ simply by $C$.

If $C$ is a holomorphic curve in $\mathbb{R} \times Y$, if $\gamma$ is an embedded Reeb orbit, and if $k$ is a positive integer, then a "positive end of $C$ at $\gamma$ of multiplicity $k$ " is an end of $C$ which is asymptotic to $\mathbb{R}$ cross the $k$-fold iterate of $\gamma$ as $s \rightarrow \infty$. This means that the end can be parametrized by $[0, \infty) \times S^{1}$ with coordinates $\tau, t$, with the almost complex structure $j$ sending $\partial_{\tau}$ to $\partial_{t}$, such that if on this end we write $u(\tau, t)=(s(\tau, t), y(\tau, t))$ with $s(\tau, t) \in \mathbb{R}$ and $y(\tau, t) \in Y$, then $\lim _{\tau \rightarrow \infty} s(\tau, \cdot)=\infty$, and $\lim _{\tau \rightarrow \infty} y(\tau, \cdot)$ is a reparametrization of the $k$-fold iterate of $\gamma$. A "negative end" is defined analogously but with $s \rightarrow-\infty$.

If $\alpha=\left\{\left(\alpha_{i}, m_{i}\right)\right\}$ and $\beta=\left\{\left(\beta_{j}, n_{j}\right)\right\}$ are two ECH generators, let $\mathcal{M}(\alpha, \beta)$ denote the moduli space of holomorphic curves in $\mathbb{R} \times Y$ with positive ends at $\alpha_{i}$ of total multiplicity $m_{i}$, negative ends at $\beta_{j}$ of total multiplicity $n_{j}$, and no other ends. If $C \in \mathcal{M}(\alpha, \beta)$, then the projection of $C$ to $Y$ has a well-defined relative homology class $[C] \in H_{2}(Y, \alpha, \beta)$. We write $I(C):=I(\alpha, \beta,[C])$.

The key nontrivial property of the ECH index is that if $C \in \mathcal{M}(\alpha, \beta)$ is not multiply covered, then

$$
\operatorname{ind}(C) \leq I(C)-2 \delta(C) .
$$

Here ind $(C)$ denotes the Fredholm index of $C$; if $J$ is generic, then $\mathcal{M}(\alpha, \beta)$ is a manifold near $C$ of dimension $\operatorname{ind}(C)$; see Dragnev [6]. Also, $\delta(C)$ is a nonnegative integer which is zero if and only if $C$ is embedded. The index inequality (2-5) was proved in a simpler setting in [13, Theorem 1.7] and is proved in the present setting in [14, Theorem 4.15] with the help of [22].

Another useful fact, which is a special case of [14, Theorem 5.1], is that if $C$ and $C^{\prime}$ are two holomorphic curves whose images in $\mathbb{R} \times Y$ do not have a common irreducible 
component, then

$$
I\left(C \cup C^{\prime}\right) \geq I(C)+I\left(C^{\prime}\right)+2 C \cdot C^{\prime} .
$$

Here $C \cdot C^{\prime}$ denotes the algebraic intersection number of $C$ and $C^{\prime}$, a nonnegative integer which is zero if and only if $C$ and $C^{\prime}$ are disjoint.

The above two inequalities imply the following classification of (possibly multiply covered) holomorphic curves with low ECH index when the almost complex structure $J$ is generic.

Notation 2.4 Any holomorphic curve $C \in \mathcal{M}(\alpha, \beta)$ can be uniquely written as $C=$ $C_{0} \cup C_{1}$, where $C_{0}$ and $C_{1}$ are holomorphic curves such that the image of $C_{0}$ is a union of $\mathbb{R}$-invariant cylinders in $\mathbb{R} \times Y$, while no component of $C_{1}$ maps to an $\mathbb{R}$-invariant cylinder.

Lemma 2.5 Suppose $J$ is generic, let $\alpha$ and $\beta$ be ECH generators, and let $C$ be in $\mathcal{M}(\alpha, \beta)$. Write $C=C_{0} \cup C_{1}$ as above. Then:

(a) $I(C) \geq 0$, with equality if and only if $C=C_{0}$.

(b) If $I(C) \in\{1,2\}$, then $C_{0}$ and $C_{1}$ are disjoint in $\mathbb{R} \times Y ; C_{1}$ is embedded in $\mathbb{R} \times Y$; and $\operatorname{ind}\left(C_{1}\right)=I\left(C_{1}\right)=I(C)$.

Proof This follows from the inequalities (2-5) and (2-6), as explained eg in [17, Proposition 7.15].

\subsection{The differential}

The differential

$$
\text { д: } C_{*}(Y, \lambda, \Gamma) \longrightarrow C_{*-1}(Y, \lambda, \Gamma)
$$

is defined as follows. Fix a generic almost complex structure $J$. If $\alpha$ is an ECH generator with $[\alpha]=\Gamma$, define

$$
\partial \alpha:=\sum_{\beta} \sum_{\substack{C \in \mathcal{M}(\alpha, \beta) / \mathbb{R} \\ I(C)=1}} \varepsilon(C) \cdot \beta .
$$

Here the first sum is over ECH generators $\beta$ with $[\beta]=\Gamma$. In the second sum, two curves $C=C_{0} \cup C_{1}$ and $C^{\prime}=C_{0}^{\prime} \cup C_{1}^{\prime}$ in $\mathcal{M}(\alpha, \beta)$ are considered equivalent whenever $C_{1}=C_{1}^{\prime}$. The $\mathbb{R}$ action on $\mathcal{M}(\alpha, \beta)$ is given by translation of the $\mathbb{R}$ coordinate on $\mathbb{R} \times Y$. Finally, $\varepsilon(C) \in\{ \pm 1\}$ is a sign, which depends on some additional choices described below. However making different choices to define the signs will result in 
isomorphic chain complexes. It is shown in [17, Lemma 7.19 and Theorem 7.20] that $\partial$ is well defined and $\partial^{2}=0$.

To determine the signs $\varepsilon(C)$, one orients all moduli spaces of non-multiply covered holomorphic curves (with the ends at positive hyperbolic orbits ordered and with no end of even multiplicity at a negative hyperbolic orbit ${ }^{1}$ ) by choosing "coherent orientations" of the relevant deformation operators with the conventions in [18, Section 9]. The quotients of such moduli spaces by the $\mathbb{R}$ action are then oriented using the " $\mathbb{R}$ direction first" convention. One also fixes an ordering of the set of all positive hyperbolic embedded Reeb orbits. Finally, given a curve $C=C_{0} \cup C_{1}$ with $I(C)=1$, writing $C_{1} \in \mathcal{M}\left(\alpha^{\prime}, \beta^{\prime}\right)$, we define $\varepsilon(C)$ to be the orientation of the point $C_{1} \in \mathcal{M}\left(\alpha^{\prime}, \beta^{\prime}\right) / \mathbb{R}$. This orientation is well defined thanks to our assumption that no hyperbolic orbit appears in an ECH generator with multiplicity greater than one.

We denote the homology of this chain complex by $\operatorname{ECH}_{*}(Y, \lambda, \Gamma)$. Although the differential $\partial$ depends on $J$, it turns out that the homology of the chain complex does not. This follows from the comparison with Seiberg-Witten Floer homology below. We also expect that one could prove this directly with holomorphic curves by a generalization of the proof in $[17 ; 18]$ that $\partial^{2}=0$.

\subsection{The U map}

There is also a degree -2 chain map

$$
U: C_{*}(Y, \lambda, \Gamma) \longrightarrow C_{*-2}(Y, \lambda, \Gamma) .
$$

The definition of $U$ was sketched in [16, Section 12], and we give more details here.

To define $U$, fix a generic almost complex structure $J$ and make the choices described above that are needed to define the signs in the differential $\partial$. Also fix a point $y \in Y$ which is not on any Reeb orbit. If $\alpha$ and $\beta$ are ECH generators, define $\mathcal{M}_{y}(\alpha, \beta)$ to be the moduli space of pairs $(u, z)$ where $u:(C, j) \rightarrow(\mathbb{R} \times Y, J)$ is an element of $\mathcal{M}(\alpha, \beta)$, and $z \in C$ is a marked point with $u(z)=(0, y)$. Finally, if $\alpha$ is an ECH generator, define

$$
U \alpha:=\sum_{\beta} \sum_{\substack{C \in \mathcal{M}_{y}(\alpha, \beta) \\ I(C)=2}} \varepsilon(C) \cdot \beta .
$$

Here the sign $\varepsilon(C)$ is defined as follows. Write $C=C_{0} \cup C_{1}$ as usual, and write $C_{1} \in \mathcal{M}\left(\alpha^{\prime}, \beta^{\prime}\right)$. Recall from Lemma $2.5(\mathrm{~b})$ that $C_{1}$ is embedded in $\mathbb{R} \times Y$, and

\footnotetext{
${ }^{1}$ For holomorphic curves with even multiplicity ends at negative hyperbolic orbits, one needs to further choose "asymptotic markings" of such ends in order to orient the moduli spaces.
} 
$\mathcal{M}\left(\alpha^{\prime}, \beta^{\prime}\right)$ is a 2 -dimensional manifold near $C_{1}$. Let $\left(v_{1}, v_{2}\right)$ be a positively oriented basis for $T_{C_{1}} \mathcal{M}\left(\alpha^{\prime}, \beta^{\prime}\right)$. The tangent vectors $v_{1}, v_{2}$ determine elements $w_{1}, w_{2}$ of the normal bundle to $C_{1}$ at $(0, y)$. A standard transversality argument shows that if $J$ is generic, then $w_{1}, w_{2}$ are necessarily linearly independent. We then define $\varepsilon(C)$ to be +1 if $\left(w_{1}, w_{2}\right)$ is a positively oriented basis for $N_{(0, y)} C_{1}$, and -1 otherwise.

Lemma 2.6 Suppose $J$ is generic. Then:

(a) $U$ is well defined.

(b) Suppose $C \in \mathcal{M}(\alpha, \beta)$ contributes to $U$, and write $C=C_{0} \cup C_{1}$ as usual. Then $C_{1}$ is embedded and connected and $\operatorname{ind}\left(C_{1}\right)=I\left(C_{1}\right)=2$.

Proof Note that if $J$ is generic, then:

(i) The elements of $\mathcal{M}_{y}(\alpha, \beta)$ with $I=2$ are isolated points in $\mathcal{M}_{y}(\alpha, \beta)$.

(ii) $(0, y)$ is not in the image of any holomorphic curve with $I=1$.

Condition (i) follows from the transversality that was discussed in the previous paragraph. Condition (ii) for generic $J$ follows from Lemma 2.5(b) together with our assumption that $y$ is not on any Reeb orbit. Assume that $J$ is generic so that (i) and (ii) hold.

(a) We need to show that if $\alpha$ and $\beta$ are ECH generators, then the set of holomorphic curves $C \in \mathcal{M}_{y}(\alpha, \beta)$ with $I(C)=2$ is finite. Suppose to the contrary that $C^{(n)}$ is an infinite sequence of distinct such curves for $n=1,2, \ldots$ By [17, Lemma 7.23], we can replace the sequence $C^{(n)}$ with a subsequence that converges in the sense of [3] to either a curve in $\mathcal{M}_{y}(\alpha, \beta)$, or a "broken" curve in which two of the levels have $I=1$ and the remaining levels have $I=0$. These cases are impossible by conditions (i) and (ii) above, respectively.

(b) All except the connectedness follows from Lemma 2.5(b). If $C_{1}$ is disconnected, then it follows from Lemma 2.5 and the inequality (2-6) that $C_{1}$ has two components and each component has $I=1$. This contradicts condition (ii) above.

One can then show that $U$ is a chain map:

$$
\partial U=U \partial .
$$

The idea of the proof is to count the ends of moduli spaces of $I=3$ curves with marked points mapping to $(0, y)$. The details are a straightforward modification of the proof that $\partial^{2}=0$ in $[17 ; 18]$, because in the analysis, curves with a marked point constraint 
behave essentially the same way as curves with index one less and no marked point constraint.

Moreover, up to chain homotopy, $U$ does not depend on the choice of $y \in Y$. To prove this, let $y^{\prime} \in Y$ be another point not on any Reeb orbit, and let $U^{\prime}$ denote the corresponding degree -2 chain map. Choose a path $\eta$ in $Y$ from $y$ to $y^{\prime}$ (here we are using the assumption that $Y$ is connected). Then counting $I=1$ curves with marked points mapping to the path $\{0\} \times \eta$ in $\mathbb{R} \times Y$, using the same signs as in the definition of $\partial$, defines a degree -1 map $K$ such that

$$
\partial K+K \partial=U-U^{\prime} .
$$

To prove this last equation, one counts ends and boundary points of the moduli space of $I=2$ curves with marked points mapping to $\{0\} \times \eta$, using the exact same gluing analysis as in the proof that $\partial^{2}=0$.

In conclusion, we obtain a well-defined map on homology

$$
U: \mathrm{ECH}_{*}(Y, \lambda, \Gamma) \longrightarrow \mathrm{ECH}_{*-2}(Y, \lambda, \Gamma) .
$$

Again, the comparison with Seiberg-Witten theory shows that this does not depend on $J$, and we expect that this can also be shown directly using holomorphic curves.

\subsection{Relation with Seiberg-Witten Floer homology}

It is shown in [24] that embedded contact homology is isomorphic to a version of Seiberg-Witten Floer homology as defined by Kronheimer and Mrowka [19]. The precise statement is that for each $\Gamma \in H_{1}(Y)$, there is an isomorphism

$$
\mathrm{ECH}_{*}(Y, \lambda, \Gamma) \simeq \widetilde{H M}_{*}(-Y, \mathfrak{s}(\xi)+\operatorname{PD}(\Gamma))
$$

of relatively $\mathbb{Z} / d\left(c_{1}(\xi)+2 \operatorname{PD}(\Gamma)\right)$-graded abelian groups ${ }^{2}$. Here $\mathfrak{s}(\xi)$ denotes the spin-c structure associated to the oriented 2-plane field $\xi$ as in [19, Section 28].

It is further shown in [25] that, at least up to signs, the isomorphism (2-8) interchanges the map $U$ in (2-7) with the map

$$
U_{\dagger}: \widetilde{H M}_{*}(-Y, \mathfrak{s}(\xi)+\operatorname{PD}(\Gamma)) \longrightarrow \widetilde{H M}_{*-2}(-Y, \mathfrak{s}(\xi)+\operatorname{PD}(\Gamma))
$$

defined in [19].

The above equivalence, together with known properties of $\widetilde{H M}$, implies the following facts about ECH which we will need.

\footnotetext{
${ }^{2}$ The right hand side of (2-8) is replaced by the canonically isomorphic group $\widehat{H M}^{-*}(Y, \mathfrak{s}(\xi)+\mathrm{PD}(\Gamma))$ in [24].
} 
Proposition 2.7 (Finiteness) $\quad$ (a) For each $\Gamma \in H_{1}(Y)$ and for each grading $*$, the group $\mathrm{ECH}_{*}(Y, \lambda, \Gamma)$ is finitely generated.

(b) There are only finitely many $\Gamma \in H_{1}(Y)$ such that $\mathrm{ECH}_{*}(Y, \lambda, \Gamma)$ is nonzero.

Proof The corresponding facts about $\widetilde{H M}_{*}$ are proved in [19, Lemma 22.3 .3 and Proposition 3.1.1].

Proposition 2.8 (Torsion spin-c structures) Let $\Gamma \in H_{1}(Y)$ and suppose that $c_{1}(\xi)+$ $2 \operatorname{PD}(\Gamma) \in H^{2}(Y ; \mathbb{Z})$ is torsion, so that $\mathrm{ECH}_{*}(Y, \lambda, \Gamma)$ is relatively $\mathbb{Z}$-graded. Then:

(a) $\operatorname{ECH}_{*}(Y, \lambda, \Gamma)$ is zero if the grading $*$ is sufficiently small.

(b) $\mathrm{ECH}_{*}(Y, \lambda, \Gamma)$ is nonzero for an infinite set of gradings $*$.

(c) If the grading $*$ is sufficiently large then the $U$ map (2-7) is an isomorphism.

Proof The corresponding properties of $\widetilde{H M}$ are proved as follows. Part (a) follows from the definition of $\widetilde{H M}$ in [19]. Part (b) is proved in [19, Cor. 35.1.4]. Part (c) is a consequence of [19, Lemma 33.3.9].

\section{More about the holomorphic curves in ECH}

We now give some more detailed information which we will need concerning the structure of the holomorphic curves that contribute to the $U$ map in $\mathrm{ECH}$. (Similar results hold for the curves that contribute to the ECH differential $\partial$, but will not be needed here.) Throughout this section fix $(Y, \lambda)$ as in Section 2, and also fix a generic almost complex structure $J$ on $\mathbb{R} \times Y$ as needed to define $\mathrm{ECH}$.

\subsection{Possible multiplicities of the ends}

We begin by recalling some restrictions on the multiplicities of the ends of holomorphic curves that contribute to $U$.

If $\gamma$ is an embedded elliptic Reeb orbit, and if $\tau$ is a trivialization of $\left.\xi\right|_{\gamma}$, then $\tau$ is homotopic to a trivialization with respect to which the linearized Reeb flow on the contact planes along $\gamma$ is rotation by angle $e^{2 \pi i \theta}$ for some real number $\theta$, which we call the monodromy angle of $\gamma$ with respect to $\tau$. Our standing assumption that all Reeb orbits are nondegenerate implies that $\theta$ is irrational. Changing the trivialization $\tau$ shifts $\theta$ by an integer.

If $\theta$ is an irrational number, define $S_{\theta}$ to be the set of positive integers $q$ such that $\left\lceil q^{\prime} \theta\right\rceil / q^{\prime}>\lceil q \theta\rceil / q$ for all $q^{\prime} \in\{1, \ldots, q-1\}$. That is, $q \in S_{\theta}$ if and only if $\theta$ is better 
approximated from above by a rational number with denominator $q$ than by a rational number with any smaller denominator. The set $S_{\theta}$ depends only on the equivalence class of $\theta$ in $\mathbb{R} / \mathbb{Z}$.

Lemma 3.1 Let $C$ be a holomorphic curve that contributes to $U$, and write $C=$ $C_{0} \cup C_{1}$ as usual. Let $\gamma$ be an embedded elliptic Reeb orbit with monodromy angle $\theta$ with respect to some trivialization. Then:

- If $C_{1}$ has a positive end at $\gamma$ of multiplicity $m$, then $m \in S_{-\theta}$.

- If $C_{1}$ has a negative end at $\gamma$ of multiplicity $m$, then $m \in S_{\theta}$.

Proof By Lemma 2.6(b), the curve $C_{1}$ is not multiply covered and has $\operatorname{ind}\left(C_{1}\right)=$ $I\left(C_{1}\right)$. The conclusions of the lemma are then part of the necessary conditions for equality in the index inequality (2-5); see [13, Theorem 1.7] or [14, Theorem 4.15]. $\square$

We now show that the allowable multiplicities have "density zero".

Notation 3.2 If $A$ is a subset of the positive integers, define the density of $A$ to be

$$
d(A):=\lim _{N \rightarrow \infty} \frac{1}{N}|A \cap\{1, \ldots, N\}|,
$$

if this limit exists.

Lemma 3.3 Let $\theta$ be an irrational number. Then $S_{\theta}$ has density zero.

Proof Write the elements of $S_{\theta}$ in increasing order as $q_{1}, q_{2}, \ldots$ It is enough to show that as $i \rightarrow \infty$, the differences $q_{i+1}-q_{i}$ are nondecreasing and tend to infinity. In fact these differences are some of the elements of $S_{-\theta}$, in increasing order, each repeated some finite number of times. One can prove this by noting that the fractions $\lceil q \theta\rceil / q$ for $q \in S_{\theta}$ and $\lfloor q \theta\rfloor / q$ for $q \in S_{-\theta}$ are the semiconvergents in the continued fraction expansion of $\theta$, and using some basic facts about continued fractions.

\subsection{Embeddedness in the 3-manifold}

The non- $\mathbb{R}$-invariant components of the holomorphic curves counted by the $U$ map are embedded in $\mathbb{R} \times Y$. Using arguments going back to Hofer, Wysocki and Zehnder [10; 11] and developed further by Siefring [21] and Wendl [26], one can show that under certain circumstances the projections of these curves to $Y$ are also embeddings, and the corresponding moduli spaces of holomorphic curves locally give a foliation of $Y$. In particular, we will need the following proposition. Some more general criteria for $3-$ dimensional embeddedness and foliations are discussed in $[21 ; 26]$. 
Proposition 3.4 Assume that $J$ is generic. Let $C$ be a holomorphic curve that contributes to $U$, and decompose $C=C_{0} \cup C_{1}$ as usual. Suppose that $C_{1}$ has genus zero, all ends of $C_{1}$ are at elliptic Reeb orbits, and $C_{1}$ does not have two positive ends or two negative ends at the same Reeb orbit. Then:

(a) The projection of $C_{1}$ to the three-manifold $Y$ is an embedding. Moreover the projections to $Y$ of the holomorphic curves in the same moduli space component as $C_{1}$ give a foliation of some subset of $Y$.

(b) $C_{1}$ does not have both a positive end and a negative end at the same Reeb orbit.

Proof The proof has seven steps.

Step 1 Let $C$ be a non- $\mathbb{R}$-invariant connected holomorphic curve. We begin by recalling the asymptotic behavior of an end of $C$ at an embedded Reeb orbit $\gamma$; for details, see Hofer, Wysocki and Zehnder [9], Siefring [22] and Hutchings and Taubes [18].

By rescaling we may assume that $\gamma$ is parametrized by $S^{1}=\mathbb{R} / \mathbb{Z}$. The asymptotic operator associated to $\gamma$ is the operator

$$
L_{\gamma}: C^{\infty}\left(\gamma^{*} \xi\right) \longrightarrow C^{\infty}\left(\gamma^{*} \xi\right)
$$

defined by

$$
L_{\gamma}:=J \nabla_{t}^{R}
$$

where $t$ denotes the $S^{1}$ coordinate and $\nabla^{R}$ denotes the connection on $\gamma^{*} \xi$ given by the linearized Reeb flow. More explicitly, choose a complex linear, symplectic trivialization $\tau$ of $\gamma^{*} \xi$; then in this trivialization,

$$
L_{\gamma}=\sqrt{-1} \frac{d}{d t}+S(t)
$$

where $S(t)$ is a symmetric $2 \times 2$ matrix. If $k$ is a positive integer, let $\gamma^{k}$ denote the $k$-fold iterate of $\gamma$, ie the pullback of $\gamma$ to $\mathbb{R} / k \mathbb{Z}$. Then the asymptotic operator associated to $\gamma^{k}$ is given in the above trivialization by

$$
L_{\gamma^{k}}=\sqrt{-1} \frac{d}{d \widetilde{t}}+S(\pi(\widetilde{t}))
$$

where $\tilde{t}$ denotes the $\mathbb{R} / k \mathbb{Z}$ coordinate and $\pi: \mathbb{R} / k \mathbb{Z} \rightarrow \mathbb{R} / \mathbb{Z}$ denotes the projection. Now identify a tubular neighborhood of $\gamma$ with $S^{1} \times D$, where $D$ is a disk in $\mathbb{C}$, such that the derivative of this identification agrees with $\tau$. A positive end of $C$ at $\gamma$ of multiplicity $k$ is then described by a map

$$
\begin{aligned}
{[R, \infty) \times \mathbb{R} / k \mathbb{Z} } & \longrightarrow \mathbb{R} \times S^{1} \times D, \\
(s, \tilde{t}) & \longmapsto(s, \pi(\tilde{t}), \varphi(s, \tilde{t})) .
\end{aligned}
$$


Moreover the function $\varphi$ satisfies

$$
\varphi(s, \tilde{t})=e^{-\lambda s}(v(\tilde{t})+\rho(s, \tilde{t}))
$$

where $v: S^{1} \rightarrow \mathbb{C}$ is a nonzero eigenfunction of the asymptotic operator $L_{\gamma^{k}}$ with eigenvalue $\lambda>0$, while $\rho(s, \widetilde{t})$ and all of its derivatives decay exponentially as $s \rightarrow \infty$. A negative end of $C$ at $\gamma$ is similarly described by

$$
\varphi(s, \tilde{t})=e^{-\lambda s}(v(\tilde{t})+\rho(s, \tilde{t}))
$$

for $s \in(-\infty, R]$, where $v$ is a nonzero eigenfunction of $L_{\gamma^{k}}$ with eigenvalue $\lambda<0$, and the function $\rho(s, \tilde{t})$ and all of its derivatives decay exponentially as $s \rightarrow-\infty$.

Note that the eigenfunction $v$ in (3-2) or (3-3) can never vanish, by Equation (3-1) and the uniqueness of solutions to ODE's. Furthermore, as shown in [10], if $\gamma$ is elliptic with monodromy $\theta$ with respect to $\tau$, then for a positive end $v$ has winding number at most

$$
\operatorname{wind}(v) \leq\lfloor k \theta\rfloor
$$

while for a negative end $v$ has winding number at least

$$
\operatorname{wind}(v) \geq\lceil k \theta\rceil
$$

Step 2 We now deduce an important inequality. Suppose that the holomorphic curve $C$ is connected, immersed and non- $\mathbb{R}$-invariant. Let $\psi$ denote the section of the normal bundle to $C$ given by the projection of $\partial / \partial s$, where $s$ denotes the $\mathbb{R}$ coordinate on $\mathbb{R} \times Y$. A calculation using the above asymptotic formulas and winding bounds along with similar winding bounds for the hyperbolic ends (cf [10]) shows that the algebraic count of zeroes of $\psi$ is finite and satisfies

$$
2 \# \psi^{-1}(0) \leq 2 g(C)-2+\operatorname{ind}(C)+h_{+}(C),
$$

where $h_{+}(C)$ denotes the number of ends of $C$ at positive hyperbolic orbits (or at negative hyperbolic orbits with even multiplicity).

Step 3 Now write $C:=C_{1}$. Lemma 2.6 implies that $C$ is connected, embedded in $\mathbb{R} \times Y$ and has Fredholm index $\operatorname{ind}(C)=2$. Thanks to our hypotheses and the fact that $\operatorname{ind}(C)=2$, the right hand side of (3-6) equals zero. On the other hand, because $\psi$ satisfies a linear PDE with the same symbol as a Cauchy-Riemann equation, all zeroes of $\psi$ have positive multiplicity. Consequently $\psi$ has no zeroes, and it follows that the projection of $C$ to $Y$ is at least an immersion. 
Step 4 Let $v$ denote the asymptotic eigenfunction associated to an end of $C$ at $\gamma$ with multiplicity $k$. We claim that there does not exist a solution to the equation

$$
v(\tilde{t})=r v(\tilde{t}+l), \quad r>0, \tilde{t} \in \mathbb{R} / k \mathbb{Z}, l \in\{1, \ldots, k-1\} .
$$

If a solution to the above equation exists, then it follows from (3-1) and the uniqueness of solutions to ODE's that $r=1$ and $v$ is the pullback of an eigenfunction of $L_{\gamma^{k^{\prime}}}$ where $k^{\prime}<k$ is a divisor of $k$. But this cannot happen because the winding number wind $(v)$ is relatively prime to $k$. To prove this last fact, one notes that the winding bounds (3-4) and (3-5) are sharp here, eg because equality holds in (3-6), and then applies Lemma 3.1 .

Step 5 For $\sigma>0$, let $C(\sigma)$ denote the holomorphic curve in $\mathbb{R} \times Y$ obtained from $C$ by translation in the $\mathbb{R}$ direction by distance $\sigma$.

Claim There exists $R>0$ such that if $\sigma>0$ is sufficiently small, then any intersection of $C$ with $C(\sigma)$ has $|s|<R$.

Proof There is an obvious bijection between the ends of $C$ and those of $C(\sigma)$. Two noncorresponding ends of $C$ and $C(\sigma)$ cannot intersect where $\sigma$ is small and $|s|$ is large by the last of our hypotheses. To show that an end of $C$ cannot intersect the corresponding end of $C(\sigma)$ where $\sigma$ is small and $|s|$ is large, one uses the asymptotic formulas (3-2) and (3-3) together with Step 4, as follows. For say a positive end, it is enough to show that the function $\varphi$ in (3-2) cannot admit a solution to the equation

$$
\varphi(s, \tilde{t})=\varphi\left(s+\sigma, \tilde{t}^{\prime}\right)
$$

where $s$ is large and $\sigma>0$ and $\pi(\tilde{t})=\pi\left(\tilde{t}^{\prime}\right)$. We consider Equation (3-7) in polar coordinates. By Step 4, if $s$ is large then the two sides of (3-7) cannot have the same angular coordinate except when $\tilde{t}=\tilde{t}^{\prime}$. But then the two sides of (3-7) cannot have the same radial coordinate, because when $s$ is large the derivative of the radial coordinate of $\varphi(s, \widetilde{t})$ with respect to $s$ is negative by (3-2).

It follows from the above claim that $C$ is disjoint from $C(\sigma)$ when $\sigma>0$ is sufficiently small. Otherwise we could take a sequence of intersections $\left\{x_{n}\right\}$ of $C$ with $C\left(\sigma_{n}\right)$ where $\sigma_{n} \rightarrow 0$, and by the claim we could pass to a convergent subsequence. The limit of this subsequence would then be a zero of $\psi$, contradicting Step 3 .

Step 6 We now complete the proof of part (a). To prove that the projection of $C$ to $Y$ is an embedding, it is enough to show that $C$ is disjoint from $C(\sigma)$ for all $\sigma>0$. More generally, to prove all of part (a) it is enough to show that if $C^{\prime} \neq C$ is any 
holomorphic curve in the same moduli space component as $C$, then $C$ and $C^{\prime}$ are disjoint.

It follows from the detailed asymptotics in [22] that $C$ and $C^{\prime}$ have only finitely many intersections. By [13, Lemma 8.5], the algebraic count of intersections of $C$ and $C^{\prime}$ is given by

$$
C \cdot C^{\prime}=Q_{\tau}(C)+\ell_{\tau}\left(C, C^{\prime}\right) .
$$

Here $\tau$ is a trivialization of $\xi$ over all the Reeb orbits at which $C$ has ends, and $\ell_{\tau}\left(C, C^{\prime}\right)$ denotes the "asymptotic linking number" of $C$ and $C^{\prime}$ with respect to $\tau$, defined in [13, Section 8.2] or [14, Section 2.7].

The asymptotic linking number is bounded from above by

$$
\ell_{\tau}\left(C, C^{\prime}\right) \leq \sum_{\gamma \in P_{+}} k\lfloor k \theta\rfloor-\sum_{\gamma \in P_{-}} k\lceil k \theta\rceil .
$$

Here the first sum is over the Reeb orbits $\gamma$ at which $C$ has a positive end, the second sum is over the Reeb orbits $\gamma$ at which $C$ has a negative end, and in each summand, $\theta$ denotes the monodromy angle of $\gamma$ with respect to $\tau$ and $k$ denotes the multiplicity of the corresponding end of $C$. The inequality (3-8) is a special case of a linking bound which is proved in a simpler situation in [13, Lem. 6.9] and which follows in the present case by the asymptotic analysis in [22]. Thus we obtain an upper bound on the algebraic intersection number:

$$
C \cdot C^{\prime} \leq Q_{\tau}(C)+\sum_{\gamma \in P_{+}} k\lfloor k \theta\rfloor-\sum_{\gamma \in P_{-}} k\lceil k \theta\rceil .
$$

The right hand side of (3-9) is a topological invariant of $C$ which does not depend on $C^{\prime}$. If $C^{\prime}=C(\sigma)$ where $\sigma>0$ is small, then it follows from the asymptotics discussed above that the inequality (3-8), and hence the inequality (3-9), is sharp. On the other hand we know from Step 5 that $C \cdot C^{\prime}=0$ in this case. Thus (3-9) says that $C \cdot C^{\prime} \leq 0$ for all $C^{\prime}$. It follows by intersection positivity that $C$ and $C^{\prime}$ are disjoint for all $C^{\prime}$.

Step 7 We now prove part (b). Suppose $\gamma$ is an embedded elliptic Reeb orbit at which $C$ has both a positive and a negative end. Let $T$ denote the boundary of a small tubular neighborhood of $\gamma$. Let $\tau$ be a trivialization of $\xi$ over $\gamma$ and identify $T \simeq S^{1} \times S^{1}$ compatibly with this trivialization, where the first $S^{1}$ factor is identified with $\gamma$. Let $\theta$ denote the monodromy angle of $\gamma$ with respect to $\tau$.

It follows from the above asymptotic formulas and winding bounds that the projection of the positive end of $C$ to $Y$ intersects $T$ transversely in a circle representing a homology class $\left(q_{+}, p_{+}\right) \in H_{1}\left(T^{2}\right)$, such that $q_{+}$is the multiplicity of the end and $p_{+} / q_{+}<\theta$. Likewise, the projection of the negative end of $C$ to $Y$ intersects $T$ 
transversely in a circle with homology class $\left(q_{-}, p_{-}\right)$satisfying $p_{-} / q_{-}>\theta$. Since $p_{+} / q_{+} \neq p_{-} / q_{-}$, the positive and negative circles in $T$ must intersect, contradicting part (a).

\subsection{Euler characteristic}

We next recall from $[14$, Section 6$]$ a variant of the ECH index, denoted by $J_{0}$, which bounds the negative Euler characteristic of holomorphic curves, similarly to the way that the ECH index $I$ bounds the Fredholm index in (2-5).

If $\alpha=\left\{\left(\alpha_{i}, m_{i}\right)\right\}$ and $\beta=\left\{\left(\beta_{j}, n_{j}\right)\right\}$ are ECH generators with $[\alpha]=[\beta]$, and if $Z \in H_{2}(Y, \alpha, \beta)$, one defines

(3-10) $J_{0}(\alpha, \beta, Z):=-c_{\tau}(Z)+Q_{\tau}(Z)+\sum_{i} \sum_{k=1}^{m_{i}-1} \mathrm{CZ}_{\tau}\left(\alpha_{i}^{k}\right)-\sum_{j} \sum_{k=1}^{n_{j}-1} \mathrm{CZ}_{\tau}\left(\beta_{j}^{k}\right)$.

Here $\tau$ is a trivialization of $\xi$ over the Reeb orbits $\alpha_{i}$ and $\beta_{j}$; one can check that $J_{0}$, like $I$, does not depend on $\tau$, even though the individual terms in its definition do. Also, like the ECH index, $J_{0}$ is additive in the sense that

$$
J_{0}(\alpha, \beta, Z)+J_{0}(\beta, \gamma, W)=J_{0}(\alpha, \gamma, Z+W) .
$$

If $C \in \mathcal{M}(\alpha, \beta)$ is a holomorphic curve, we write $J_{0}(C):=J_{0}(\alpha, \beta,[C])$.

We now have the following bound on topological complexity in terms of $J_{0}$.

Lemma 3.5 Let $C \in \mathcal{M}(\alpha, \beta)$ be a holomorphic curve that contributes to $U$. Write $C=C_{0} \cup C_{1}$ as usual. Then

$$
J_{0}(C) \geq 2 g\left(C_{1}\right)-2+\sum_{i}\left(2 n_{i}^{+}+t_{i}^{+}-1\right)+\sum_{j}\left(2 n_{j}^{-}+t_{j}^{-}-1\right) .
$$

Here $g\left(C_{1}\right)$ denotes the genus of $C_{1} ; n_{i}^{+}$denotes the number of positive ends of $C_{1}$ at $\alpha_{i}$ and $n_{j}^{-}$denotes the number of negative ends of $C_{1}$ at $\beta_{j}^{-} ; t_{i}^{+}$is 1 if the image of $C_{0}$ contains $\mathbb{R} \times \alpha_{i}$ and 0 otherwise; and $t_{j}^{-}$is 1 if the image of $C_{0}$ contains $\mathbb{R} \times \beta_{j}$ and 0 otherwise ${ }^{3}$.

Proof Since $C_{1}$ is embedded by Lemma 2.6(b), one can apply [14, Proposition 6.9] to obtain

$$
J_{0}\left(C_{1}\right) \geq 2 g\left(C_{1}\right)-2+\sum_{i: n_{i}^{+}>0}\left(2 n_{i}^{+}-1\right)+\sum_{j: n_{j}^{-}>0}\left(2 n_{j}^{-}-1\right) .
$$

\footnotetext{
${ }^{3}$ In fact equality holds in (3-12) here. One can show this by the arguments in [14], or by a more direct calculation using the necessary conditions for equality in (2-5). However we will not need this.
} 
Also [14, Proposition 6.14] implies that

$$
J_{0}(C) \geq J_{0}\left(C_{0}\right)+J_{0}\left(C_{1}\right)+\sum_{i: n_{i}^{+}>0} t_{i}^{+}+\sum_{j: n_{j}^{-}>0} t_{j}^{-} .
$$

Finally, it follows from the definition of $J_{0}$ that

$$
J_{0}\left(C_{0}\right)=0 .
$$

Combining (3-13), (3-14) and (3-15) proves the lemma.

It follows immediately from the inequality (3-12) that

$$
J_{0}(C) \geq-1,
$$

since all of the summands in the sums over $i$ and $j$ are nonnegative and $C_{1}$ has at least one end. We also deduce the following criterion for recognizing holomorphic cylinders in a certain situation which will arise later.

Lemma 3.6 Let $\gamma_{1}$ and $\gamma_{2}$ be distinct embedded elliptic Reeb orbits and let $\alpha=$ $\gamma_{1}^{m_{1}} \gamma_{2}^{m_{2}}$ and $\alpha^{\prime}=\gamma_{1}^{m_{1}^{\prime}} \gamma_{2}^{m_{2}^{\prime}}$ with $m_{1}, m_{2}, m_{1}^{\prime}, m_{2}^{\prime} \neq 0$. Suppose $C \in \mathcal{M}\left(\alpha, \alpha^{\prime}\right)$ contributes to $U$, and write $C=C_{0} \cup C_{1}$ as usual. Assume that $C_{1}$ has ends at both $\gamma_{1}$ and $\gamma_{2}$. Then:

(a) $J_{0}(C) \geq 2$.

(b) If $J_{0}(C)=2$, then $C_{1}$ is a cylinder.

Proof (a) In this situation the inequality (3-12) can be rewritten as

$$
\frac{1}{2} J_{0}(C) \geq g\left(C_{1}\right)-3+\sum_{i=1}^{2}\left(n_{i}^{+}+n_{i}^{-}+T_{i}\right),
$$

where $T_{i}$ is defined to be 1 if the image of $C_{0}$ contains $\mathbb{R} \times \gamma_{i}$ and 0 otherwise. Since $C_{1}$ has ends at both $\gamma_{1}$ and $\gamma_{2}$, we have $n_{i}^{+}+n_{i}^{-} \geq 1$. Also, since $m_{i}, m_{i}^{\prime} \neq 0$, it follows that

$$
T_{i}=0 \quad \Longrightarrow \quad n_{i}^{+}, n_{i}^{-} \geq 1
$$

So in all cases we have

$$
n_{i}^{+}+n_{i}^{-}+T_{i} \geq 2
$$

Putting (3-19) into (3-17) gives $J_{0}(C) \geq 2$.

(b) By the above, if $J_{0}(C)=2$ then $g\left(C_{1}\right)=0$ and $n_{i}^{+}+n_{i}^{-}+T_{i}=2$ for $i=1,2$. If $T_{1}=T_{2}=1$, then it follows immediately that $C_{1}$ is a cylinder so we are done. If some $T_{i}=0$, then $n_{i}^{+}=n_{i}^{-}=1$ by (3-18). But this contradicts Proposition 3.4(b). $\square$ 


\section{Contact 3-manifolds with all Reeb orbits elliptic}

We now prove Theorem 1.2. The proof occupies Sections 4.1-4.7 below. We then prove Theorem 1.3 in Section 4.8.

\subsection{Initial input from Seiberg-Witten theory}

Throughout the proof of Theorem 1.2, fix a closed oriented connected 3-manifold $Y$ with a contact form $\lambda$ such that all Reeb orbits are nondegenerate and elliptic. Also fix a generic almost complex structure on $\mathbb{R} \times Y$ in order to define the $\mathrm{ECH}$ chain complex.

We begin by using Propositions 2.7 and 2.8 from Seiberg-Witten theory to make some basic observations which will be used repeatedly below.

Lemma 4.1 Under our assumption that all Reeb orbits are nondegenerate and elliptic:

(a) All differentials in the ECH chain complex vanish.

(b) If $\gamma$ is a Reeb orbit then $[\gamma]$ is torsion in $H_{1}(Y)$.

(c) $c_{1}(\xi)$ is torsion in $H^{2}(Y ; \mathbb{Z})$.

Proof (a) Equation (2-4) and our assumption that all Reeb orbits are elliptic imply that the relative index $I(\alpha, \beta)$ is always even, so the differential vanishes.

(b) If $\gamma$ is an embedded Reeb orbit, then for each nonnegative integer $m$, the ECH generator $\gamma^{m}$ represents a nonzero class in $\mathrm{ECH}_{*}(Y, \lambda, m[\gamma])$. If $[\gamma]$ is not torsion, then the homology classes $\{m[\gamma]\}_{m=0,1, \ldots}$ give infinitely many $\Gamma$ for which $\mathrm{ECH}_{*}(Y, \lambda, \Gamma)$ is nonzero, contradicting Proposition 2.7(b).

(c) Since $c_{1}(\xi)$ is divisible by 2 in $H^{2}(Y ; \mathbb{Z})$, there exists $\Gamma \in H_{1}(Y)$ such that $c_{1}(\xi)+2 \mathrm{PD}(\Gamma)=0$. By Proposition 2.8(b), there exists an admissible orbit set $\alpha=\left\{\left(\alpha_{i}, m_{i}\right)\right\}$ with $[\alpha]=\sum_{i} m_{i}\left[\alpha_{i}\right]=\Gamma$. It then follows from part (b) that $c_{1}(\xi)$ is torsion.

This lemma simplifies the computation of the ECH index as follows. Let $\alpha$ and $\beta$ be two ECH generators with $[\alpha]=[\beta] \in H_{1}(Y)$, let $\tau$ be a trivialization of $\xi$ over the Reeb orbits in $\alpha$ and $\beta$, and let $Z \in H_{2}(Y, \alpha, \beta)$. Then by Equation (2-1), the relative first Chern class $c_{\tau}(Z)$ depends only on $\alpha, \beta$ and $\tau$, so we can denote it by $c_{\tau}(\alpha, \beta)$. Likewise, by Equation (2-2), the relative intersection pairing $Q_{\tau}(Z)$ depends only on $\alpha, \beta$ and $\tau$, so we can denote it by $Q_{\tau}(\alpha, \beta)$. 


\subsection{Computing the ECH index}

We now compute the ECH index of all of the ECH generators. For simplicity, let us temporarily assume that all Reeb orbits are nullhomologous; we will remove this assumption in Section 4.7.

Let $\gamma_{1}, \ldots, \gamma_{n}$ denote the distinct embedded Reeb orbits. (At this point in the argument there could be infinitely many of them, in which case $n$ should be replaced by $\infty$ in the summations below.) The ECH generators have the form $\gamma_{1}^{m_{1}} \cdots \gamma_{n}^{m_{n}}$ where $m_{1}, \ldots, m_{n}$ are nonnegative integers. This product notation is shorthand for the orbit set $\left\{\left(\gamma_{i}, m_{i}\right) \mid i=1, \ldots, n ; m_{i} \neq 0\right\}$.

Since $c_{1}(\xi)$ is torsion and all of the Reeb orbits are nullhomologous, the relative index on ECH has a unique refinement to an absolute index which assigns to each generator $\alpha$ an integer $I(\alpha)$ such that $I(\alpha, \beta)=I(\alpha)-I(\beta)$ and $I(\varnothing)=0$. To describe this integer, fix a trivialization $\tau$ of the contact structure $\xi$ over the $\gamma_{i}$ 's. The index of an ECH generator $\alpha=\gamma_{1}^{m_{1}} \cdots \gamma_{n}^{m_{n}}$ is then given by

$$
I(\alpha)=c_{\tau}(\alpha)+Q_{\tau}(\alpha)+\sum_{i=1}^{n} \sum_{k=1}^{m_{i}} \mathrm{CZ}_{\tau}\left(\gamma_{i}^{k}\right) .
$$

Here $c_{\tau}(\alpha)$ is shorthand for $c_{\tau}(\alpha, \varnothing)$, and $Q_{\tau}(\alpha)$ is shorthand for $Q_{\tau}(\alpha, \varnothing)$.

To make this more explicit, define $c_{i}:=c_{\tau}\left(\gamma_{i}, \varnothing\right)$ and $Q_{i}:=Q_{\tau}\left(\gamma_{i}, \varnothing\right)$. Also, for $i \neq j$ define $Q_{i j}:=Q_{\tau}\left(Z_{i}, Z_{j}\right)$ where $Z_{i} \in H_{2}\left(Y, \gamma_{i}, \varnothing\right)$ and $Z_{j} \in H_{2}\left(Y, \gamma_{j}, \varnothing\right)$. In fact it follows from the definition of $Q$ in $[13 ; 14]$ that $Q_{i j}$ does not depend on $Z_{i}, Z_{j}$, or $\tau$ and is just the linking number of $\gamma_{i}$ and $\gamma_{j}$. Finally, let $\theta_{i}$ denote the monodromy angle of $\gamma_{i}$ with respect to $\tau$; see Section 3.1. Since $c_{\tau}$ is linear in the relative homology class, we have

$$
c_{\tau}(\alpha)=\sum_{i=1}^{n} m_{i} c_{i}
$$

Also $Q_{\tau}$ is quadratic in the sense that

$$
Q_{\tau}(\alpha)=\sum_{i=1}^{n} m_{i}^{2} Q_{i}+\sum_{i \neq j} m_{i} m_{j} Q_{i j}
$$

(see [13, Equation (68)] or [14, Equation (3.11)]). Finally, the Conley-Zehnder terms are given explicitly by

$$
\mathrm{CZ}_{\tau}\left(\gamma_{i}^{k}\right)=2\left\lfloor k \theta_{i}\right\rfloor+1
$$


To simplify the resulting expression for the ECH index, define

$$
\eta_{i}:=\left(c_{i}-Q_{i}+1\right) / 2, \quad \phi_{i}:=Q_{i}+\theta_{i} .
$$

We then obtain

$$
\frac{1}{2} I(\alpha)=\sum_{i=1}^{n} m_{i} \eta_{i}+\sum_{i<j} m_{i} m_{j} Q_{i j}+\sum_{i=1}^{n} \sum_{k=1}^{m_{i}}\left\lfloor k \phi_{i}\right\rfloor .
$$

We remark that the quantities $\eta_{i}$ and $\phi_{i}$ are natural to consider because they do not depend on $\tau$ (even though $c_{i}, Q_{i}$ and $\theta_{i}$ do). In fact $Q_{i}-c_{i}$ agrees with a familiar quantity from contact topology, namely the self-linking number of the transverse knot $\gamma_{i}$ (see eg Geiges [7, Section 3.5.2]); while $\phi_{i}$ is some irrational number.

Example 4.2 Suppose $Y$ is an ellipsoid

$$
\left\{\left(z_{1}, z_{2}\right) \mid \frac{\left|z_{1}^{2}\right|}{a_{1}}+\frac{\left|z_{2}\right|^{2}}{a_{2}}=1\right\} \subset \mathbb{C}^{2},
$$

where $a_{1} / a_{2}$ is irrational, with the standard contact form. Here there are exactly two Reeb orbits, both elliptic, given by the circles $z_{1}=0$ and $z_{2}=0$. One can calculate that $\eta_{1}=\eta_{2}=Q_{12}=\phi_{1} \phi_{2}=1$. It is an exercise to deduce from Equation (4-2) that there is exactly one generator of each nonnegative even index ${ }^{4}$, as there should be since $Y \simeq S^{3}$ and the Seiberg-Witten Floer homology $\widetilde{H M}$ of $S^{3}$ is known to have one generator in each nonnegative even degree. It is interesting to compare this example with the characterization of ellipsoids in terms of linearized contact homology in [2].

\subsection{Proof that there are exactly two embedded orbits}

We now prove that $n=2$. The idea is to use Proposition 2.8 and argue that if $n=1$ then there are not enough generators in a given index range, and if $n \geq 3$ then there are too many.

By estimating $k \phi_{i}-1 \leq\left\lfloor k \phi_{i}\right\rfloor \leq k \phi_{i}$ in Equation (4-2), we find that the ECH index is approximated by

$$
I(\alpha)=\bar{Q}\left(m_{1}, \ldots, m_{n}\right)+O\left(m_{1}+\cdots+m_{n}\right),
$$

\footnotetext{
${ }^{4}$ The solution to the exercise is to consider the line $L$ in the plane with slope $-\phi_{1}$ passing through the point $\left(m_{1}, m_{2}\right)$. The right side of (4-2) is then the number of lattice points in the triangle consisting of the line $L$ and the coordinate axes (including lattice points on the boundary), minus 1 . As one moves the line $L$ up and to the right, keeping its slope fixed, one hits all of the lattice points in the positive quadrant in succession, and each lattice point has index 2 greater than the previous one.
} 
where $\bar{Q}$ denotes the quadratic form

$$
\bar{Q}\left(m_{1}, \ldots, m_{n}\right):=\sum_{i=1}^{n} m_{i}^{2} \phi_{i}+\sum_{i \neq j} m_{i} m_{j} Q_{i j} .
$$

Also recall that the numbers $\phi_{i}$ are irrational. It follows that $n \geq 2$, because if $n<2$, then the number of ECH generators with index $\leq k$ is bounded from above by a linear function of $\sqrt{k}$ plus a constant, so there are not enough generators of large index to satisfy Proposition 2.8(b),(c).

On the other hand, it follows from (4-3) that there is a constant $c$ such that

$$
I(\alpha) \leq c\left(m_{1}^{2}+\cdots+m_{n}^{2}+1\right) .
$$

Hence the number of ECH generators with index $\leq k$ is bounded from below by a constant times $(k-c)^{n / 2}$. But by Propositions 2.7(a) and 2.8(a),(c), the number of generators of index $\leq k$ is bounded from above by a linear function of $k$ plus a constant. Thus $n \leq 2$.

\subsection{A lower bound on the ECH index}

With $n=2$ proved, we now establish an additional estimate on the ECH index which will be needed later:

Lemma 4.3 There are constants $c_{1}, c_{2}>0$ such that if $\alpha=\gamma_{1}^{m_{1}} \gamma_{2}^{m_{2}}$ with $m_{1}, m_{2} \geq 0$, then

$$
I(\alpha) \geq c_{1}\left(m_{1}^{2}+m_{2}^{2}\right)-c_{2} .
$$

Proof By the estimate (4-3), if $\alpha=\gamma_{1}^{m_{1}} \gamma_{2}^{m_{2}}$ with $m_{1}, m_{2} \geq 0$ then

$$
\begin{aligned}
& I(\alpha) \geq \bar{Q}\left(m_{1}, m_{2}\right)-L\left(m_{1}, m_{2}\right), \\
& I(\alpha) \leq \bar{Q}\left(m_{1}, m_{2}\right)+L\left(m_{1}, m_{2}\right),
\end{aligned}
$$

where $L$ is a linear function. By the lower bound in (4-4), it is enough to show that $\bar{Q}\left(m_{1}, m_{2}\right)>0$ whenever $m_{1}$ and $m_{2}$ are nonnegative and not both zero. It follows from Proposition 2.8(a) and the upper bound in (4-5) that $\bar{Q}$ is nonnegative on all lines of rational slope in the quadrant $\left\{\left(m_{1}, m_{2}\right) \mid m_{1}, m_{2} \geq 0\right\}$. Therefore $\bar{Q}$ is nonnegative on the whole quadrant. Moreover $\bar{Q}$ is positive on the coordinate axes (minus the origin), because $\phi_{1}$ and $\phi_{2}$ are irrational. So we just need to rule out the case where $\bar{Q}$ is degenerate and its null space has positive slope.

In the case to be ruled out,

$$
\bar{Q}\left(m_{1}, m_{2}\right)=\phi_{1}\left(m_{1}-\lambda m_{2}\right)^{2}
$$


where $\phi_{1}, \lambda>0$. We can then complete the square in the upper bound (4-5) to obtain

$$
I(\alpha) \leq \phi_{1}\left(m_{1}-\lambda m_{2}+a_{1}\right)^{2}+a_{2} m_{1}+a_{3}
$$

where $a_{1}, a_{2}, a_{3}$ are constants. But this contradicts the linear growth in the number of ECH generators with index $\leq k$ as $k \rightarrow \infty$. To see this, note that if $a_{2} \leq 0$, then by taking lattice points near the line $m_{1}=\lambda m_{2}$ we can find infinitely many ECH generators with index bounded from above. And if $a_{2}>0$, then calculation using (4-6) shows that the number of ECH generators with index $\leq k$ grows as at least $k^{3 / 2}$.

We remark that in Example 4.2, the quadratic form $\bar{Q}$ is degenerate, and its null space has negative slope.

\subsection{Existence of a holomorphic cylinder}

We now put everything together to prove:

Lemma 4.4 Let $\gamma_{1}$ and $\gamma_{2}$ denote the distinct embedded Reeb orbits as above. Then there exist positive integers $m_{1}, m_{2}$ and an $I=2$ cylinder $C$ in $\mathcal{M}\left(\gamma_{1}^{m_{1}}, \gamma_{2}^{m_{2}}\right)$ or $\mathcal{M}\left(\gamma_{2}^{m_{2}}, \gamma_{1}^{m_{1}}\right)$ or $\mathcal{M}\left(\gamma_{1}^{m_{1}} \gamma_{2}^{m_{2}}, \varnothing\right)$.

Proof By Proposition 2.8, if $k_{0}$ is a sufficiently large integer, then there exist ECH generators of index $2 k_{0}$, and the $U$ map is an isomorphism in all degrees higher than $2 k_{0}$. Let $\alpha(0)$ be a generator with $I(\alpha(0))=2 k_{0}$. By induction on $k$ we can find a sequence of $\mathrm{ECH}$ generators

$$
\left\{\alpha(k)=\gamma_{1}^{m_{1}(k)} \gamma_{2}^{m_{2}(k)}\right\}
$$

indexed by $k \geq 0$ such that for each $k \geq 1$, there exists

$$
C(k) \in \mathcal{M}(\alpha(k), \alpha(k-1))
$$

which contributes to $U$. In particular,

$$
I(\alpha(k))=2\left(k_{0}+k\right) .
$$

Now we consider positive integers $k$ such that the curves $C(k)$ have certain desirable properties. Let

$$
\begin{aligned}
& A_{1}:=\left\{k>0 \mid m_{i}(k), m_{i}(k-1) \neq 0 \text { for } i=1,2\right\}, \\
& A_{2}:=\left\{k>0 \mid m_{i}(k) \notin S_{-\theta_{i}} \text { and } m_{i}(k-1) \notin S_{\theta_{i}} \text { for } i=1,2\right\}, \\
& A_{3}:=\left\{k>0 \mid C(k)_{1} \text { has ends at both } \gamma_{1} \text { and } \gamma_{2}, \text { or } J_{0}(C(k)) \geq 3\right\}, \\
& A_{4}:=\left\{k>0 \mid J_{0}(C(k))=2\right\} .
\end{aligned}
$$


Here in the definition of $A_{3}$ we are decomposing $C(k)=C(k)_{0} \cup C(k)_{1}$ as usual. It is enough to show that $A_{1} \cap A_{3} \cap A_{4}$ is nonempty, because if $k \in A_{1} \cap A_{3} \cap A_{4}$ then Lemma 3.6(b) is applicable to the curve $C(k)$, and $C(k)_{1}$ is the cylinder we are seeking. To prove that $A_{1} \cap A_{3} \cap A_{4}$ is nonempty, we will show that for each $j=1,2,3,4$ the set $A_{j}$ has density 1 . (The fact that $A_{2}$ has density 1 will be used in the proofs that $A_{3}$ and $A_{4}$ do.)

To show that $A_{1}$ has density 1 , note that since $I(\alpha(k))$ grows linearly with $k$; see Equation (4-7), it follows from Lemma 4.3 that

(*) There is a constant $c$ such that for each positive integer $N$, the $N+1$ points $\left(m_{1}(0), m_{2}(0)\right), \ldots,\left(m_{1}(N), m_{2}(N)\right)$ are all contained in a ball of radius $c(\sqrt{N}+1)$ centered at the origin.

Consequently $\left|\{1, \ldots, N\} \backslash A_{1}\right|$ grows as at most $\sqrt{N}$, so $A_{1}$ has density 1 . Likewise, (*) and Lemma 3.3 imply that $A_{2}$ has density 1 .

We now show that $A_{3}$ has density 1 . Since $A_{1}$ and $A_{2}$ have density 1 , it is enough to show that $\left(A_{1} \cap A_{2}\right) \backslash A_{3}$ has density 0 . Suppose $k \in\left(A_{1} \cap A_{2}\right) \backslash A_{3}$ and let $C:=C(k)$. Since $k \notin A_{3}$, without loss of generality $C_{1}$ has ends only at $\gamma_{1}$, and $J_{0}(C) \leq 2$. These two conditions, together with the assumption $k \in A_{1}$ and Lemma 3.5 , imply that

$$
g\left(C_{1}\right)+n_{1}^{+}+n_{1}^{-}+T_{1} \leq 3 .
$$

Here $n_{1}^{+}$denotes the number of positive ends of $C_{1}$ at $\gamma_{1} ; n_{1}^{-}$denotes the number of negative ends of $C_{1}$ at $\gamma_{1}$; and $T_{1}$ is defined to be 1 if the image of $C_{0}$ contains $\mathbb{R} \times \gamma_{1}$ and zero otherwise. Note also that $n_{1}^{+} \geq 1$ since any holomorphic curve of the type we are considering must have at least one positive end. We then deduce from the above inequality that at least one of the following cases holds:

(i) $C_{1}$ has no negative end.

(ii) The image of $C_{0}$ does not contain $\mathbb{R} \times \gamma_{1}$, and $C_{1}$ has exactly one positive end or exactly one negative end.

(iii) $C_{1}$ is a cylinder with both positive and negative ends.

Now case (ii) is impossible by our assumption that $k \in A_{2}$ and Lemma 3.1. And case (iii) is impossible by Proposition 3.4(b). So to complete the proof that $A_{3}$ has density 1 , it is enough to show that case (i) only happens for $k$ in a set of density zero. If case (i) holds, let $\widetilde{C}_{1}$ denote the union of $C_{1}$ with the part of $C_{0}$ that maps to $\mathbb{R} \times \gamma_{1}$. By the superadditivity of the ECH index in Equation (2-6) and Lemma 2.6(b), we have $2=I(C) \geq I\left(\widetilde{C}_{1}\right) \geq I\left(C_{1}\right)=2$, so

$$
I\left(\widetilde{C}_{1}\right)=I\left(C_{1}\right)=2 .
$$


That is, writing $m:=m_{1}(k)$ and $m^{\prime}:=m_{1}(k-1)$, we have

$$
I\left(\gamma_{1}^{m}\right)-I\left(\gamma_{1}^{m^{\prime}}\right)=I\left(\gamma_{1}^{m-m^{\prime}}\right)=2 .
$$

By Equation (4-2), this is equivalent to

$$
\sum_{k=1}^{m-m^{\prime}}\left\lfloor\left(m^{\prime}+k\right) \phi_{1}\right\rfloor=\sum_{k=1}^{m-m^{\prime}}\left\lfloor k \phi_{1}\right\rfloor=1-\eta_{1}\left(m-m^{\prime}\right) .
$$

Recall that $\phi_{1}>0$. Now the left equality requires that $m^{\prime} \leq\left\lceil 1 / \phi_{1}\right\rceil$, because otherwise each term in the left sum would be greater than the corresponding term in the right sum. And the right equality can only hold for finitely many values of $m-m^{\prime}$, because the left side is approximated by a quadratic function of $m-m^{\prime}$ while the right side is a linear function of $m-m^{\prime}$. We conclude that case (i) can only hold for finitely many pairs $\left(m_{1}(k), m_{1}(k-1)\right)$, and by $(*)$ again this can only happen for $k$ in a set of density zero.

To show that $A_{4}$ has density 1 , we first show that $J_{0}$ is "close" to $I$. In the present situation, the relative index $J_{0}$, just like $I$, can be uniquely refined to an absolute index which associates to each ECH generator an integer, such that $J_{0}(\alpha, \beta)=J_{0}(\alpha)-J_{0}(\beta)$ and $J_{0}(\varnothing)=0$. Similarly to (4-2), we find that if $\alpha=\gamma_{1}^{m_{1}} \gamma_{2}^{m_{2}}$, then

$$
\frac{1}{2} J_{0}(\alpha)=\sum_{i=1}^{2} m_{i}\left(1-\eta_{i}\right)+m_{1} m_{2} Q_{12}+\sum_{i=1}^{2} \sum_{k=1}^{m_{i}-1}\left\lfloor k \phi_{i}\right\rfloor-\frac{1}{2} \#\left\{i \mid m_{i} \neq 0\right\} .
$$

Subtracting this from Equation (4-2), we obtain

$$
I(\alpha)-J_{0}(\alpha)=\sum_{i=1}^{2} m_{i}\left(4 \eta_{i}-2\right)+2 \sum_{i=1}^{2}\left\lfloor m_{i} \phi_{i}\right\rfloor+\#\left\{i \mid m_{i} \neq 0\right\} .
$$

This equation implies that there is a constant $c$ such that

$$
\left|J_{0}(\alpha)-I(\alpha)\right| \leq c\left(m_{1}+m_{2}\right) .
$$

It follows using $(*)$ that for any $\varepsilon>0$, if $N$ is sufficiently large then

$$
\left|J_{0}(\alpha(N))-I(\alpha(N))\right| \leq \varepsilon N .
$$

Then by Equation (4-7), for any $\varepsilon>0$, if $N$ is sufficiently large then

$$
J_{0}(\alpha(N))-J_{0}(\alpha(0)) \leq(2+\varepsilon) N .
$$


On the other hand, by the additivity of $J_{0}$, we have

$$
J_{0}(\alpha(N))-J_{0}(\alpha(0))=\sum_{k=1}^{N} J_{0}(C(k)) .
$$

Now it follows from Lemma 3.6(a) that $J_{0}(C(k)) \geq 2$ for all $k$ in the set $A_{1} \cap A_{3}$, which has density 1 . Also recall from (3-10) and (3-16) that $J_{0}(C(k))$ is always an integer and always at least -1 . Combining this with (4-8) and (4-9), we conclude that $J_{0}(C(k))=2$ for $k$ in a set of density 1 .

\subsection{Existence of a genus one Heegaard splitting}

To complete the proof of Theorem 1.2 (modulo our temporary simplifying assumption that all Reeb orbits are nullhomologous), we now show that $Y$ has a genus 1 Heegaard splitting such that the Reeb orbits $\gamma_{1}$ and $\gamma_{2}$ are the core circles of the corresponding solid tori.

By Lemma 4.4, without loss of generality there exists a cylinder $C \in \mathcal{M}\left(\gamma_{1}^{m_{1}}, \gamma_{2}^{m_{2}}\right)$, for some $m_{1}, m_{2} \neq 0$, with ECH index $I(C)=2$. (It makes no difference in the argument below if both ends of $C$ are positive.) Let $\mathcal{M}_{C}$ denote the component of $\mathcal{M}\left(\gamma_{1}^{m_{1}}, \gamma_{2}^{m_{2}}\right)$ containing $C$. Let $\pi: \mathbb{R} \times Y \rightarrow Y$ denote the projection. By Proposition 3.4(a), $\pi(C)$ is embedded in $Y$, and the projections to $Y$ of the cylinders in $\mathcal{M}_{C}$ comprise a foliation of some subset of $Y$. Since all ECH generators have even index, it follows from the compactness theorem in [13, Theorem 1.8] or [17, Lemma 7.23] that the moduli space $\mathcal{M}_{C} / \mathbb{R}$ is compact. Therefore the projections of the cylinders in $\mathcal{M}_{C}$ foliate all of $Y \backslash\left(\gamma_{1} \cup \gamma_{2}\right)$, and $\mathcal{M}_{C} / \mathbb{R} \simeq S^{1}$.

For each $i=1,2$ let $T_{i} \subset Y$ denote a torus given by the boundary of a small closed tubular neighborhood $N_{i}$ of $\gamma_{i}$. It follows from the asymptotics for holomorphic curves reviewed in Section 3.2 that the end at $\gamma_{i}$ of each holomorphic curve in $\mathcal{M}_{C}$, when projected to $Y$, intersects the torus $T_{i}$ transversely in a single circle. These circles then foliate the torus $T_{i}$.

It follows that if $C^{\prime} \in \mathcal{M}_{C}$, then $\pi\left(C^{\prime}\right)$ intersects $N_{i}$ only in a single half-closed cylinder corresponding to the end of $C^{\prime}$ at $\gamma_{i}$. Thus $\pi\left(C^{\prime}\right)$ intersects $Y \backslash \operatorname{int}\left(N_{1} \cup N_{2}\right)$ in a closed cylinder. For each element of $\mathcal{M}_{C} / \mathbb{R}$, we can choose a diffeomorphism of this closed cylinder with $S^{1} \times[1,2]$, sending the corresponding circle in $T_{i}$ to $S^{1} \times\{i\}$. There is no obstruction to choosing these diffeomorphisms to be smooth functions on $\mathcal{M}_{C} / \mathbb{R} \simeq S^{1}$, so that they combine to give a diffeomorphism

$$
\varphi: S^{1} \times S^{1} \times[1,2] \stackrel{\simeq}{\longrightarrow} Y \backslash \operatorname{int}\left(N_{1} \cup N_{2}\right)
$$


identifying $S^{1} \times S^{1} \times\{i\}$ with $T_{i}$. Now

and

$$
\begin{aligned}
& N_{1} \cup \varphi\left(S^{1} \times S^{1} \times[1,3 / 2]\right) \\
& N_{2} \cup \varphi\left(S^{1} \times S^{1} \times[3 / 2,2]\right)
\end{aligned}
$$

are solid tori in $Y$ which give the desired Heegaard splitting.

\subsection{Removing the simplifying assumption}

We now prove Theorem 1.2 without assuming that all Reeb orbits are nullhomologous. As in Section 4.2, let $\gamma_{1}, \ldots, \gamma_{n}$ denote the distinct embedded Reeb orbits. We know from Lemma 4.1(b) that these represent torsion homology classes, so for each $i=$ $1, \ldots, n$ let $l_{i}$ denote the smallest positive integer such that $\gamma_{i}^{l_{i}}$ is nullhomologous. If $m_{1}, \ldots, m_{n}$ are nonnegative integers such that $l_{i}$ divides $m_{i}$ for each $i$, then $\gamma_{1}^{m_{1}} \ldots \gamma_{n}^{m_{n}}$ is a nullhomologous orbit set. As such it has a well-defined absolute ECH index. To compute this, similarly to Section 4.2, let $\tau$ be a trivialization of $\xi$ over the $\gamma_{i}$ 's, let $c_{i}:=c_{\tau}\left(\gamma_{i}^{l_{i}}, \phi\right) / l_{i}$, let $Q_{i}:=Q_{\tau}\left(\gamma_{i}^{l_{i}}, \phi\right) / l_{i}^{2}$, for $i \neq j$ let $Q_{i, j}$ denote the linking number of $\gamma_{i}^{l_{i}}$ and $\gamma_{j}^{l_{j}}$ divided by $l_{i} l_{j}$, and let $\theta_{i}$ denote the monodromy angle of $\gamma_{i}$ with respect to $\tau$. Then just as in (4-2),

$$
I\left(\gamma_{1}^{m_{1}} \cdots \gamma_{n}^{m_{n}}\right)=\sum_{i=1}^{n}\left(c_{i} m_{i}+Q_{i} m_{i}^{2}\right)+\sum_{i \neq j} m_{i} m_{j} Q_{i j}+\sum_{i=1}^{n} \sum_{k=1}^{m_{i}}\left(2\left\lfloor k \theta_{i}\right\rfloor+1\right)
$$

In fact, the linear property of $c_{\tau}$ and the quadratic property of $Q_{\tau}$ imply that the index formula (4-10) is valid for any nullhomologous ECH generator $\gamma_{1}^{m_{1}} \cdots \gamma_{n}^{m_{n}}$, not just one in which each $m_{i}$ is a multiple of $l_{i}$.

We can approximate the index formula (4-10) by

$$
I\left(\gamma_{1}^{m_{1}} \cdots \gamma_{n}^{m_{n}}\right)=\bar{Q}\left(m_{1}, \ldots, m_{n}\right)+O\left(m_{1}+\cdots+m_{n}\right),
$$

where the quadratic form $\bar{Q}$ is defined by

$$
\bar{Q}\left(m_{1}, \ldots, m_{n}\right):=\sum_{i=1}^{n}\left(Q_{i}+\theta_{i}\right) m_{i}^{2}+\sum_{i \neq j} m_{i} m_{j} Q_{i j} .
$$

The same argument as in Section 4.3 then shows that $n=2$. Also the same argument as in Section 4.4 shows that Lemma 4.3 still holds for nullhomologous ECH generators. One just needs to divide each estimate on the number of nullhomologous ECH generators by the density of the lattice

$$
\left\{\left(m_{1}, m_{2}\right) \in \mathbb{Z}^{2} \mid m_{1}\left[\gamma_{1}\right]+m_{2}\left[\gamma_{2}\right]=0 \in H_{1}(Y)\right\} .
$$


Lemma 4.4 then holds by the same argument, using a sequence of nullhomologous ECH generators provided by Proposition 2.8. Finally, the argument in Section 4.6 produces a genus 1 Heegaard splitting just as before. Note that $Y \not \neq S^{1} \times S^{2}$ because $\left[\gamma_{1}\right]$ and $\left[\gamma_{2}\right]$ are torsion. So $Y$ is a lens space, and the proof of Theorem 1.2 is complete.

\subsection{The theorem on three Reeb orbits}

Proof of Theorem 1.3 Let $Y$ be a closed oriented connected 3-manifold with a contact form $\lambda$ such that all Reeb orbits are nondegenerate, and fix a generic almost complex structure on $\mathbb{R} \times Y$ to define $\mathrm{ECH}$.

First note that if all Reeb orbits are hyperbolic, then there must be infinitely many distinct embedded Reeb orbits. Otherwise by definition the ECH chain complex would have only finitely many generators, contradicting Proposition 2.8 .

So by Theorem 1.2, it is enough to rule out the case where there are exactly two embedded Reeb orbits, one elliptic and one hyperbolic. Suppose that this holds and denote these orbits by $e$ and $h$ respectively. The ECH generators are now $e^{m} h^{n}$ where $m \geq 0$ and $n \in\{0,1\}$.

By Proposition 2.8(b), there exists $\Gamma \in H_{1}(Y)$ such that there are infinitely many ECH generators $\alpha$ with $[\alpha]=\Gamma$. It follows that the homology class $[e] \in H_{1}(Y)$ is torsion.

The homology class $[h]$ is also torsion. Proof: If $[h]$ is not torsion then the ECH differential vanishes identically as in Lemma 4.1(a). Since $[e]$ is torsion, it follows that $\mathrm{ECH}_{*}(Y, \lambda, \Gamma)$ is infinitely generated for both $\Gamma=0$ and $\Gamma=[h]$. Since $[h]$ is not torsion, at least one of these classes $\Gamma$ must have the property that $c_{1}(\xi)+2 \operatorname{PD}(\Gamma) \epsilon$ $H^{2}(Y ; \mathbb{Z})$ is not torsion. For such a class $\Gamma$ there are only finitely many possible values of the grading on $\mathrm{ECH}_{*}(Y, \lambda, \Gamma)$, and now Proposition 2.7(a) gives a contradiction.

Since all Reeb orbits represent torsion homology classes, the cohomology class $c_{1}(\xi) \in$ $H^{2}(Y ; \mathbb{Z})$ is then also torsion, as in Lemma 4.1(c).

If $[h] \neq 0$, then the same argument as in Section 4.3 gives a contradiction, by showing that $\mathrm{ECH}_{*}(Y, \lambda, 0)$ does not have enough generators in a given index range in order to be consistent with Proposition 2.8. If $[h]=0$ then this argument also works, because $I\left(e^{m} h\right)$ differs from $I\left(e^{m}\right)$ by a linear function of $m$.

\section{The Weinstein conjecture for stable Hamiltonian structures}

This section is devoted to the proof of the main theorem, Theorem 1.1. 


\subsection{Warmup cases}

We first prove the theorem in the special cases when $f$ vanishes either nowhere or everywhere.

If $f$ is nowhere vanishing, then $Y$ is a contact manifold (with the opposite orientation if $f<0$ ), so the theorem in this case is just the Weinstein conjecture which we already know (even if $Y$ is a $T^{2}$-bundle over $S^{1}$ ).

Now suppose $f=0$, so that the 1 -form $\lambda$ is closed. Since $\lambda \wedge \omega>0$, it follows that $[\lambda] \smile[\omega] \neq 0$ in $H^{3}(Y ; \mathbb{R})$, so $\lambda$ represents a nonzero cohomology class in $H^{1}(Y ; \mathbb{R})$. We can then add a small closed $1-$ form so as to replace $\lambda$ by a closed $1-$ form $\lambda^{\prime}$ which represents a real multiple of an integral cohomology class and still satisfies $\lambda^{\prime} \wedge \omega>0$ everywhere. Since $\left[\lambda^{\prime}\right]$ is a multiple of an integral class, there is a fiber bundle $\pi: Y \rightarrow S^{1}$ such that $\lambda^{\prime}$ is a multiple of the pullback of the volume form on $S^{1}$. Since $\lambda^{\prime} \wedge \omega>0$, it follows that $\omega$ restricts to a symplectic form on each fiber of $\pi$. Since $R$ is in the kernel of $\omega$, we deduce that $R$ is transverse to the fibers. If $F$ is a fiber, then the return map of the flow $R$ defines an orientation-preserving diffeomorphism $\phi: F \rightarrow F$, and closed orbits of $R$ are equivalent to periodic orbits of $\phi$. By replacing $\phi$ with an iterate if necessary, we may assume without loss of generality that $F$ is connected. The theorem in this case now follows from part (a) of the following lemma. Part (b) will be needed later.

Lemma 5.1 Let $F$ be a closed oriented connected surface and let $\phi: F \rightarrow F$ be an orientation-preserving diffeomorphism. Suppose that $\phi$ has only finitely many irreducible periodic orbits and that all periodic orbits are nondegenerate and elliptic ${ }^{5}$. Then:

(a) If $\phi$ has no periodic orbits, then $F$ is a torus.

(b) Otherwise $F$ is a sphere, $\phi$ has exactly two fixed points, and these are the only irreducible periodic orbits.

Proof Let $A$ denote the induced map $\phi_{*}: H_{1}(F) \rightarrow H_{1}(F)$. Since all periodic orbits are elliptic, every periodic point of $\phi$ of period $p$ counts with weight +1 in the Lefschetz fixed point formula for $\phi^{p}$. We then have the identity

$$
\frac{\operatorname{det}(1-t A)}{(1-t)^{2}}=\prod_{\gamma}\left(1-t^{p(\gamma)}\right)^{-1}
$$

\footnotetext{
${ }^{5}$ Here "nondegenerate and elliptic" means that the eigenvalues of the linearized return map are not on the real line.
} 
of formal power series in $\mathbb{Z}[[t]]$, where the product is over irreducible periodic orbits $\gamma$, and $p(\gamma)$ denotes the period of $\gamma$. This formula is a special case of the product formula for the Lefschetz zeta function (see eg [15]), and it is proved by taking the logarithmic derivative of both sides and then using the Lefschetz fixed point formula for $\phi$ and its iterates. Now we can rewrite the product formula here as

$$
\operatorname{det}(1-t A) \prod_{\gamma}\left(1-t^{p(\gamma)}\right)=(1-t)^{2} .
$$

Since by hypothesis there are only finitely many factors on the left hand side, each a polynomial, it follows that the sum of the degrees of these factors must equal 2 , ie

$$
2 g(F)+\sum_{\gamma} p(\gamma)=2 .
$$

The lemma follows immediately.

To prove Theorem 1.1 in the remaining cases, assume that $R$ has no closed orbit and that the function $f$ is sometimes zero and sometimes nonzero. We must show that $Y$ is a $T^{2}$-bundle over $S^{1}$.

\subsection{The region where $f$ is large}

Lemma 5.2 Suppose $\varepsilon \geq 0$ is a regular value of $f$. Then

$$
Y_{\geq \varepsilon}:=\{y \in Y \mid f(y) \geq \varepsilon\}
$$

is diffeomorphic to a disjoint union of copies of $T^{2} \times I$.

Proof The idea is to collapse each boundary component of $Y_{\geq \varepsilon}$ to a circle, so as to obtain a closed contact manifold with one embedded elliptic Reeb orbit for each boundary component, and then invoke Theorem 1.2. We proceed in three steps.

Step 1 We begin by choosing coordinates near the boundary of $Y_{\geq \varepsilon}$ in which the stable Hamiltonian structure has a nice form.

Fix $\delta>0$ sufficiently small so that every number in the interval $[\varepsilon, \varepsilon+\delta]$ is a regular value of $f$. Fix a component $Z$ of $f^{-1}[\varepsilon, \varepsilon+\delta]$. For $s \in[0, \delta]$, let $\Sigma_{s}$ denote the component of $f^{-1}(\varepsilon+s)$ in $Z$. Each $\Sigma_{s}$ is a torus, because the Reeb vector field $R$ is nonvanishing and tangent to $\Sigma_{s}$.

There is a unique vector field $W$ on $Z$ such that $\lambda(W)=0$ and $\omega(W, \cdot)=d f$. The vector field $W$ is tangent to each $\Sigma_{S}$ and commutes with $R$ by (1-1); and the vectors $R$ and $W$ are linearly independent at each point. 
Claim We can find smooth real-valued functions $\alpha(s), \beta(s), \gamma(s), \sigma(s)$ such that for each $s$, the vector fields $\alpha(s) R+\beta(s) W$ and $\gamma(s) R+\sigma(s) W$ are linearly independent on $\Sigma_{s}$ and have all orbits closed with period 1 .

Proof Fix $s \in[0, \delta]$. For $t \in \mathbb{R}$ let $\Phi_{R}^{t}: \Sigma_{s} \rightarrow \Sigma_{s}$ and $\Phi_{W}^{t}: \Sigma_{s} \rightarrow \Sigma_{s}$ denote the time $t$ flows of $R$ and $W$ respectively on $\Sigma_{s}$. Fix a point $p \in \Sigma_{s}$ and define a map $\phi: \mathbb{R}^{2} \rightarrow \Sigma_{s}$ by

$$
\phi(\alpha, \beta):=\Phi_{R}^{\alpha} \Phi_{W}^{\beta}(p) .
$$

Since $R$ and $W$ are linearly independent and commute, it follows that $\phi$ is a covering map and $\phi^{-1}(p)$ is a lattice in $\mathbb{R}^{2}$. Choose $(\alpha(s), \beta(s))$ and $(\gamma(s), \sigma(s))$ to be a basis for this lattice. Then $\alpha(s), \beta(s), \gamma(s), \sigma(s)$ have the required properties for our fixed $s$. These can be uniquely extended to smooth functions of $s \in[0, \delta]$ which satisfy the required properties for all $s$.

It follows from the claim that we can find coordinates $s \in[0, \delta]$ and $x_{1}, x_{2} \in \mathbb{R} / \mathbb{Z}$ on $Z$ in which

$$
\begin{gathered}
f\left(s, x_{1}, x_{2}\right)=\varepsilon+s, \\
R=a_{1}(s) \frac{\partial}{\partial x_{1}}+a_{2}(s) \frac{\partial}{\partial x_{2}} .
\end{gathered}
$$

Since $R$ is assumed to have no closed orbits, the ratio $a_{1}(s) / a_{2}(s)$ is an irrational number which does not depend on $s$. In fact $a_{1}(s)$ and $a_{2}(s)$ do not depend on $s$ either. To see this, note that since $\mathcal{L}_{R} \lambda=0$, we have

$$
\begin{aligned}
R(\lambda(\partial / \partial s)) & =\left(\mathcal{L}_{R} \lambda\right)(\partial / \partial s)+\lambda\left(\mathcal{L}_{R}(\partial / \partial s)\right) \\
& =-\lambda\left(\mathcal{L}_{\partial / \partial s} R\right) \\
& =-\lambda\left(a_{1}^{\prime}(s) \frac{\partial}{\partial x_{1}}+a_{2}^{\prime}(s) \frac{\partial}{\partial x_{2}}\right) .
\end{aligned}
$$

Since the right hand side depends only on $s$, and since the function $\lambda(\partial / \partial s)$ is bounded on $\Sigma_{s}$, it follows that the right hand side is zero. Therefore

$$
a_{1}^{\prime}(s) \frac{\partial}{\partial x_{1}}+a_{2}^{\prime}(s) \frac{\partial}{\partial x_{2}}
$$

is a multiple of $W$, and this multiple must be zero because $a_{1}(s) / a_{2}(s)$ is constant. Since $\omega(R, \cdot)=0$, we can write

$$
\omega=c(s) d s \wedge\left(a_{2} d x_{1}-a_{1} d x_{2}\right) .
$$


Note that the function $c$ depends only on $s$, because $\mathcal{L}_{R} \omega=0$ implies $R(c)=0$, and $R$ has dense orbits on each $\Sigma_{s}$. Also $c$ is nowhere vanishing, and we can choose our coordinates so that $c$ is always positive. Likewise, since $\lambda(R)=1$, we can write

$$
\lambda=b(s)\left(a_{2} d x_{1}-a_{1} d x_{2}\right)+\sigma(s) d s+\left(a_{1}^{2}+a_{2}^{2}\right)^{-1}\left(a_{1} d x_{1}+a_{2} d x_{2}\right),
$$

where $b$ and $\sigma$ depend only on $s$ because $\mathcal{L}_{R} \lambda=0$. Since $d \lambda=f \omega$, we have

$$
b^{\prime}(s)=(\varepsilon+s) c(s) .
$$

Also, changing $\lambda$ by adding the differential of some function of $s$ does not change its salient properties, so we may assume that $\sigma(s)=0$.

Step 2 We now collapse the boundary component $\Sigma_{0}$ to a circle.

To prepare for this, define a function $\rho:[0, \delta] \rightarrow \mathbb{R}$ by

$$
\rho(s)^{2}:=2(b(s)-b(0)) \text {. }
$$

Since $\varepsilon \geq 0$ and $c(s)$ is always positive, it follows from Equation (5-1) that $\rho$ is strictly increasing and smooth on $(0, \delta]$. In terms of this function we can write

$$
\lambda=\frac{1}{2} \rho^{2}\left(a_{2} d x_{1}-a_{1} d x_{2}\right)+\alpha_{1} d x_{1}+\alpha_{2} d x_{2}
$$

where $\alpha_{1}$ and $\alpha_{2}$ are constants satisfying $a_{1} \alpha_{1}+a_{2} \alpha_{2}=1$.

We next modify $\lambda$ on $Y_{\geq \varepsilon}$, without changing its salient properties, to arrange that $\alpha_{1} / \alpha_{2}$ is rational. Suppose that $\alpha_{1} / \alpha_{2}$ is irrational. The restriction map $H^{1}\left(Y_{\geq \varepsilon} ; \mathbb{Z}\right) \rightarrow$ $H^{1}\left(\Sigma_{0} ; \mathbb{Z}\right)$ is nonzero, and so there exists a closed 1 -form $\lambda_{1}$ on $Y_{\geq \varepsilon}$ such that $\left.\lambda_{1}\right|_{Z}=\beta_{1} d x_{1}+\beta_{2} d x_{2}$ for some relatively prime integers $\beta_{1}$ and $\beta_{2}$. Now consider replacing $\lambda$ by $\lambda+\tau \lambda_{1}$ where $\tau$ is a small constant. If $\tau>0$ is sufficiently small then we still have $\left(\lambda+\tau \lambda_{1}\right) \wedge \omega>0$. And since $\alpha_{1} / \alpha_{2}$ is irrational and $\beta_{1} / \beta_{2}$ is rational, it follows that $\left(\alpha_{1}+\tau \beta_{1}\right) /\left(\alpha_{2}+\tau \beta_{2}\right)$ is rational for a dense set of $\tau$. Replacing $\lambda$ by $\lambda+\tau \lambda_{1}$ multiplies the Reeb vector field by a positive function; in particular there are still no Reeb orbits.

With $\alpha_{1} / \alpha_{2}$ arranged to be rational, by an $S L_{2} \mathbb{Z}$ coordinate change we can further assume that $\alpha_{1}=0$. Now let $Y_{\geq \varepsilon}^{\prime}$ be obtained from $Y_{\geq \varepsilon}$ by declaring two points in $\Sigma_{0}$ to be equivalent whenever they have the same $x_{1}$ coordinate. The subset $Z$ of $Y_{\geq \varepsilon}$ gets collapsed to a subset $Z^{\prime}$ of $Y_{\geq \varepsilon}^{\prime}$ which is a disk cross $S^{1}$. Define the smooth structure on $Z^{\prime}$ so that $\left(\rho, x_{1}\right)$ are polar coordinates on the disk and $x_{2}$ is the $S^{1}$ coordinate. Then $\lambda$ is a smooth contact form on $Z^{\prime}$, because in the above coordinates,

$$
\lambda=\frac{1}{2} \rho^{2}\left(a_{2} d x_{1}-a_{1} d x_{2}\right)+\alpha_{2} d x_{2}
$$


is smooth, and

$$
\lambda \wedge d \lambda=\rho d \rho d x_{1} d x_{2}
$$

is nonvanishing. The boundary torus $\Sigma_{0}$ gets collapsed to a new Reeb orbit $\Sigma_{0}^{\prime}$ in $Y_{\geq \varepsilon}^{\prime}$. This Reeb orbit is elliptic with irrational monodromy angle, because $R$ has irrational slope on the tori where $\rho$ is constant. The Reeb vector field on $Y_{\geq_{\varepsilon}}^{\prime} \backslash \Sigma_{0}^{\prime}=Y_{\geq \varepsilon} \backslash \Sigma_{0}$ is unaffected.

Step 3 We now complete the proof of Lemma 5.2.

Repeating the above process finitely many times, we collapse all boundary components of $Y_{\geq \varepsilon}$ to circles, to obtain a closed contact manifold with one embedded elliptic Reeb orbit for each boundary component, all of whose iterates are nondegenerate, and no other embedded Reeb orbits. Let $X$ be a component of $Y_{\geq \varepsilon}$. It then follows from Theorem 1.2 that there are exactly two such orbits which comprise the core circles of a genus 1 Heegaard splitting of the collapsed $X$. Uncollapsing these circles back to boundary tori, we conclude that $X$ is diffeomorphic to $T^{2} \times I$.

The same argument with some different signs shows that if $\varepsilon \leq 0$ is a regular value of $f$, then

$$
Y_{\leq \varepsilon}:=\{y \in Y \mid f(y) \leq \varepsilon\}
$$

is likewise diffeomorphic to a disjoint union of copies of $T^{2} \times I$. In particular, if 0 is a regular value of $f$, then it follows that $Y$ is a union of copies of $T^{2} \times I$ glued together along their boundaries, so Theorem 1.1 is proved in this case.

\subsection{The region where $f$ is small}

We now begin the proof of Theorem 1.1 when 0 is not necessarily a regular value of $f$. Choose a small $\varepsilon>0$ such that both $\varepsilon$ and $-\varepsilon$ are regular values of $f$. Define

$$
Y_{\varepsilon}:=\{y \in Y|| f(y) \mid \leq \varepsilon\} .
$$

To prove Theorem 1.1, we will show that if $\varepsilon$ as above is sufficiently small, then $Y_{\varepsilon}$ is a disjoint union of copies of $T^{2} \times I$. The strategy for doing so is to perturb $\left.\lambda\right|_{Y_{\varepsilon}}$ to a closed form which still has positive wedge product with $\omega$, deduce that $Y_{\varepsilon}$ fibers over $S^{1}$ such that the Reeb vector field is transverse to the fibers, and then apply Lemma 5.1(b) to show that the fibers are disjoint unions of annuli.

To start, fix a metric on $Y$. Also fix a smooth function $\chi:[0,1] \rightarrow[0,1]$ such that $\chi(t)=1$ for $t<1 / 3$ and $\chi(t)=0$ for $t>2 / 3$.

Fix $\varepsilon$ as above. Choose $\delta \in(0, \varepsilon)$ sufficiently small so that all numbers in the intervals $[\varepsilon, \varepsilon+\delta]$ and $[-\varepsilon-\delta,-\varepsilon]$ are regular values of $f$. Define a $1-$ form $\lambda_{*}$ on $Y_{\varepsilon+\delta}$ as 
follows. On $Y_{\varepsilon}$ define $\lambda_{*}:=\lambda$. We now define $\lambda_{*}$ on $f^{-1}[\varepsilon, \varepsilon+\delta]$. The construction on $f^{-1}[-\varepsilon-\delta,-\varepsilon]$ is analogous and will be omitted.

Fix a component $Z$ of $f^{-1}[\varepsilon, \varepsilon+\delta]$. Recall from Section 5.2 that we can choose coordinates $s \in[0, \delta]$ and $x_{1}, x_{2} \in \mathbb{R} / \mathbb{Z}$ on $Z$, and modify $\lambda$ without changing its salient properties, so that in these coordinates

$$
\begin{aligned}
& \lambda=b(s)\left(a_{2} d x_{1}-a_{1} d x_{2}\right)+\frac{a_{1} d x_{1}+a_{2} d x_{2}}{a_{1}^{2}+a_{2}^{2}}, \\
& \omega=c(s) d s \wedge\left(a_{2} d x_{1}-a_{1} d x_{2}\right),
\end{aligned}
$$

where $c(s)>0$. We now define $\lambda_{*}$ on $Z$ by

$$
\lambda_{*}:=\left(b(0)+\chi\left(\delta^{-1} s\right)(b(s)-b(0))\left(a_{2} d x_{1}-a_{1} d x_{2}\right)+\frac{a_{1} d x_{1}+a_{2} d x_{2}}{a_{1}^{2}+a_{2}^{2}} .\right.
$$

On $Y_{\varepsilon+\delta}$ we then have

$$
d \lambda_{*}=g \omega
$$

where $g$ is a smooth function which agrees with $f$ on $Y_{\varepsilon}$ and extends by zero to a smooth function defined on all of $Y$.

\subsection{An upper bound on $g$}

We now show that there is an $\varepsilon$-independent constant $c_{0}$ such that if $\delta$ is chosen sufficiently small in the above construction then

$$
|g| \leq c_{0} \varepsilon .
$$

We just need to check this on a region $Z$ as above. It follows from the above equations that on $Z$ we have

$$
g(s)=c(s)^{-1}\left(\delta^{-1} \chi^{\prime}\left(\delta^{-1} s\right)(b(s)-b(0))+\chi\left(\delta^{-1} s\right) b^{\prime}(s)\right) .
$$

Since $b^{\prime}(s)=(\varepsilon+s) c(s)$ and we have chosen $\delta<\varepsilon$, it follows that

$$
c(s)^{-1} \chi\left(\delta^{-1} s\right) b^{\prime}(s)=\chi\left(\delta^{-1} s\right)(\varepsilon+s)<2 \varepsilon .
$$

If we further choose $\delta$ sufficiently small so that $c(t) \leq 2 c(s)$ for all $s, t \in[0, \delta]$, then we can estimate

$$
\begin{aligned}
b(s)-b(0) & =\int_{0}^{s}(\varepsilon+t) c(t) d t \\
& \leq 2 c(s)\left(\varepsilon s+s^{2} / 2\right) \\
& \leq 3 c(s) \varepsilon \delta .
\end{aligned}
$$

Putting the above two inequalities into (5-3) proves (5-2). 


\subsection{The cohomology class of $g \omega$}

We now study the cohomology class of the closed 2-form $g \omega$ on $Y$.

Let $U \subset Y$ denote the open set where $f \neq 0$. Consider the relative homology long exact sequence:

$$
\cdots \longrightarrow H_{1}(U) \longrightarrow H_{1}(Y) \longrightarrow H_{1}(Y, U) \longrightarrow \cdots
$$

Here and below all homology and cohomology is with real coefficients. Fix embedded oriented curves $\gamma_{1}, \ldots, \gamma_{n} \subset U$ that represent a basis for the kernel of the map $H_{1}(Y) \rightarrow$ $H_{1}(Y, U)$. Since the curves $\gamma_{i}$ all have positive distance from the compact set where $f=0$, there exists $d>0$ such that if $\varepsilon$ is sufficiently small then no point in $Y_{\varepsilon}$ is within distance $d$ of any point in any of the curves $\gamma_{i}$. For each $i=1, \ldots, n$, fix an $\varepsilon$-independent closed 2-form $\mu_{i}$ which represents the Poincaré dual of $\gamma_{i}$ and is supported within distance $d / 2$ of $\gamma_{i}$.

Claim There exists an $\varepsilon$-independent constant $c_{0}$ such that if $\varepsilon$ is chosen sufficiently small in the construction in Section 5.3, then there are unique real numbers $q_{1}, \ldots, q_{n}$ such that

$$
[g \omega]=\sum_{i=1}^{n} q_{i}\left[\mu_{i}\right] \in H^{2}(Y),
$$

and these satisfy

$$
\left|q_{i}\right| \leq c_{0} \varepsilon
$$

Note that $c_{0}$ here is different than in (5-2).

Proof Choose $\varepsilon$ sufficiently small so that $Y_{\varepsilon}$ does not intersect the support of the forms $\mu_{i}$. Then the Poincaré duality isomorphism $H_{1}(Y) \stackrel{\simeq}{\longrightarrow} H^{2}(Y)$ restricts to an injection

$$
\operatorname{Ker}\left(H_{1}(Y) \rightarrow H_{1}(Y, U)\right) \longrightarrow \operatorname{Ker}\left(H^{2}(Y) \rightarrow H^{2}\left(Y_{\varepsilon}\right)\right) .
$$

Since $q \omega$ restricts to an exact form on $Y_{\varepsilon}$, to prove that the numbers $q_{i}$ exist and are unique it is enough to show that the map (5-6) is surjective. To prove this surjectivity, note that any element of $\operatorname{Ker}\left(H^{2}(Y) \rightarrow H^{2}\left(Y_{\varepsilon}\right)\right)$ can be represented by a closed 2-form $\eta$ with support on $Y \backslash Y_{\varepsilon}$. Here we are assuming as usual that $\varepsilon$ and $-\varepsilon$ are regular values of $f$. Now the Poincare dual of $[\eta] \in H_{c}^{2}\left(Y \backslash Y_{\varepsilon}\right)$ is a homology class $\alpha \in H_{1}\left(Y \backslash Y_{\varepsilon}\right)$ with $\alpha \cdot S=\int_{S} \eta$ for all $S \in H_{2}\left(Y \backslash Y_{\varepsilon}, \partial Y_{\varepsilon}\right)$. Letting $\imath: Y \backslash Y_{\varepsilon} \rightarrow Y$ denote the inclusion, we then have $l_{*} \alpha \cdot S=\int_{S} \eta$ for all $S \in H_{2}(Y)$. So $l_{*} \alpha \in H_{1}(Y)$ is the Poincaré dual of $[\eta] \in H^{2}(Y)$, and $l_{*} \alpha$ maps to 0 in $H_{1}(Y, U)$ since $Y \backslash Y_{\varepsilon} \subset U$. 
To prove that $\left|q_{i}\right| \leq c_{0} \varepsilon$, for each $i=1, \ldots, n$ we can fix an $\varepsilon$-independent closed oriented embedded surface $\Sigma_{i}$ in $Y$ which has intersection number $\delta_{i j}$ with $\gamma_{j}$. Then observe that

$$
q_{i}=\int_{\Sigma_{i}} \sum_{j=1}^{n} q_{j} \mu_{j}=\int_{\Sigma_{i}} g \omega
$$

and use (5-2).

\subsection{The region where $f$ is small fibers over $S^{1}$}

We now show that if $\varepsilon$ is sufficiently small then $Y_{\varepsilon}$ fibers over $S^{1}$ so that the Reeb vector field $R$ is transverse to the fibers.

Let $\varepsilon>0$ be small enough so that $Y_{\varepsilon}$ does not intersect the support of the forms $\mu_{i}$. Let $q_{i}$ be the numbers satisfying (5-4). Since the 2-form

$$
g \omega-\sum_{i=1}^{n} q_{i} \mu_{i}
$$

is exact on $Y$, there exists a unique 1 -form $v$ such that

$$
d \nu=g \omega-\sum_{i=1}^{n} q_{i} \mu_{i}
$$

and $d^{*} v=0$ and $v$ is $L^{2}$-orthogonal to the space of harmonic 1 -forms on $Y$. It then follows from estimates on the Green's function for $d+d^{*}$, namely the fact that the singularity of the Green's function behaves as $\operatorname{dist}\left(y_{1}, y_{2}\right)^{-2}$ for any points $y_{1} \neq y_{2}$ in $Y$, that there is an $\varepsilon$-independent constant $c_{0}$ such that

$$
|v| \leq c_{0} \sup _{Y}\left|g \omega-\sum_{i=1}^{n} q_{i} \mu_{i}\right| .
$$

With (5-2) and (5-5), this implies that there is an $\varepsilon$-independent constant $c_{0}$ such that

$$
|v| \leq c_{0} \varepsilon
$$

Now the 1-form $\lambda-v$ restricts to a closed 1-form on $Y_{\varepsilon}$. By the estimate (5-7), if $\varepsilon$ is sufficiently small then $(\lambda-v) \wedge \omega>0$ everywhere. We can perturb $\lambda-v$ to a closed 1-form $\lambda^{\prime}$ on $Y_{\varepsilon}$ which represents a real multiple of an integral cohomology class in $H^{1}\left(Y_{\varepsilon} ; \mathbb{Z}\right)$ and still satisfies $\lambda^{\prime} \wedge \omega>0$. It follows as in Section 5.1 that $Y_{\varepsilon}$ fibers over $S^{1}$ with the Reeb vector field $R$ transverse to the fibers. 


\subsection{The fibers are disjoint unions of annuli}

To complete the proof of Theorem 1.1, we now show that $Y_{\varepsilon}$ is diffeomorphic to a disjoint union of copies of $T^{2} \times I$.

Let $X$ be a component of $Y_{\varepsilon}$. Let $F$ be a fiber of the fibration $X \rightarrow S^{1}$ constructed in Section 5.6, and let $\phi: F \rightarrow F$ denote the return map of the flow $R$. Closed orbits of $R$ in $X$ are equivalent to periodic orbits of $\phi$, so by assumption $\phi$ has no periodic orbits. By replacing $\phi$ by an iterate if necessary, we may assume without loss of generality that $F$ is connected.

Assume as usual that $\varepsilon$ and $-\varepsilon$ are regular values of $f$, so that the boundary of $X$ is a disjoint union of tori. Each boundary circle of $F$ lies in a boundary torus of $X$. Recall from Section 5.2 that on each boundary torus of $X$, in suitable coordinates the Reeb vector field $R$ is a constant vector field with irrational slope which does not change if we perturb $\varepsilon$.

Let $F^{\prime}$ be the closed surface obtained from $F$ by identifying two points whenever they are in the same boundary circle. Then $\phi$ descends to a diffeomorphism $\phi^{\prime}: F^{\prime} \rightarrow F^{\prime}$ with one irreducible periodic orbit $\gamma$ for each boundary torus of $X$, and no other irreducible periodic orbits. The period of $\gamma$ equals the number of boundary circles of $F^{\prime}$ on the corresponding boundary torus of $X$. It follows from the above description of $R$ near the boundary of $X$ that the orbit $\gamma$ and all of its iterates are nondegenerate and elliptic.

We now invoke Lemma 5.1(b) to conclude $F^{\prime}$ is a sphere and $\phi^{\prime}$ has two fixed points and no other irreducible periodic orbits. It follows immediately that $F$ is an annulus, so $X$ is diffeomorphic to $T^{2} \times I$ as desired. This completes the proof of Theorem 1.1.

\section{References}

[1] C Abbas, K Cieliebak, H Hofer, The Weinstein conjecture for planar contact structures in dimension three, Comment. Math. Helv. 80 (2005) 771-793 MR2182700

[2] F Bourgeois, K Cieliebak, T Ekholm, A note on Reeb dynamics on the tight 3-sphere, J. Mod. Dyn. 1 (2007) 597-613 MR2342700

[3] F Bourgeois, Y Eliashberg, H Hofer, K Wysocki, E Zehnder, Compactness results in symplectic field theory, Geom. Topol. 7 (2003) 799-888 MR2026549

[4] K Cieliebak, K Mohnke, Compactness for punctured holomorphic curves, J. Symplectic Geom. 3 (2005) 589-654 MR2235856Conference on Symplectic Topology

[5] V Colin, K Honda, Reeb vector fields and open book decompositions arXiv: 0809.5088 
[6] D L Dragnev, Fredholm theory and transversality for noncompact pseudoholomorphic maps in symplectizations, Comm. Pure Appl. Math. 57 (2004) 726-763 MR2038115

[7] H Geiges, An introduction to contact topology, Cambridge Studies in Advanced Math. 109, Cambridge University Press (2008) MR2397738

[8] H Hofer, Pseudoholomorphic curves in symplectizations with applications to the Weinstein conjecture in dimension three, Invent. Math. 114 (1993) 515-563 MR1244912

[9] H Hofer, K Wysocki, E Zehnder, Properties of pseudoholomorphic curves in symplectisations. I. Asymptotics, Ann. Inst. H. Poincaré Anal. Non Linéaire 13 (1996) 337-379 MR1395676

[10] H Hofer, K Wysocki, E Zehnder, Properties of pseudoholomorphic curves in symplectisations. II. Embedding controls and algebraic invariants, Geom. Funct. Anal. 5 (1995) 270-328 MR1334869

[11] H Hofer, K Wysocki, E Zehnder, Finite energy foliations of tight three-spheres and Hamiltonian dynamics, Ann. of Math. (2) 157 (2003) 125-255 MR1954266

[12] H Hofer, E Zehnder, Symplectic invariants and Hamiltonian dynamics, Birkhäuser Verlag, Basel (1994) MR1306732

[13] M Hutchings, An index inequality for embedded pseudoholomorphic curves in symplectizations, J. Eur. Math. Soc. 4 (2002) 313-361 MR1941088

[14] M Hutchings, The embedded contact homology index revisited, to appear in the Yashafest proceedings arXiv:0805.1240

[15] M Hutchings, Y-J Lee, Circle-valued Morse theory, Reidemeister torsion, and SeibergWitten invariants of 3-manifolds, Topology 38 (1999) 861-888 MR1679802

[16] M Hutchings, M Sullivan, Rounding corners of polygons and the embedded contact homology of $T^{3}$, Geom. Topol. 10 (2006) 169-266 MR2207793

[17] M Hutchings, C H Taubes, Gluing pseudoholomorphic curves along branched covered cylinders. I, J. Symplectic Geom. 5 (2007) 43-137 MR2371184

[18] M Hutchings, C H Taubes, Gluing pseudoholomorphic curves along branched covered cylinders. II arXiv:0705.2074

[19] P Kronheimer, T Mrowka, Monopoles and three-manifolds, New Math. Monogr. 10, Cambridge University Press (2007) MR2388043

[20] A Rechtman, Use and disuse of plugs in foliations, preprint (2008)

[21] R Siefring, Intersection theory of punctured pseudoholomorphic curves, in preparation

[22] R Siefring, The relative asymptotic behavior of pseudoholomorphic half-cylinders, to appear in Comm. Pure Appl. Math. arXiv:math/0702356

[23] C H Taubes, The Seiberg-Witten equations and the Weinstein conjecture, Geom. Topol. 11 (2007) 2117-2202 MR2350473 
[24] C H Taubes, Embedded contact homology and Seiberg-Witten Floer cohomology I-IV, preprints (2008)

[25] C H Taubes, Embedded contact homology and Seiberg-Witten Floer cohomology V, in preparation

[26] C Wendl, Punctured holomorphic curves with boundary in 3-manifolds: Fredholm theory and embeddedness, in preparation

Mathematics Department, 970 Evans Hall, University of California Berkeley, CA 94720, USA

Mathematics Department, Harvard University

Cambridge, MA 02138, USA

hutching@math.berkeley.edu, chtaubes@math.harvard.edu

Proposed: Yasha Eliashberg

Seconded: Peter Ozsvath, Leonid Polterovich
Received: 21 September 2008 Revised: 8 December 2008 